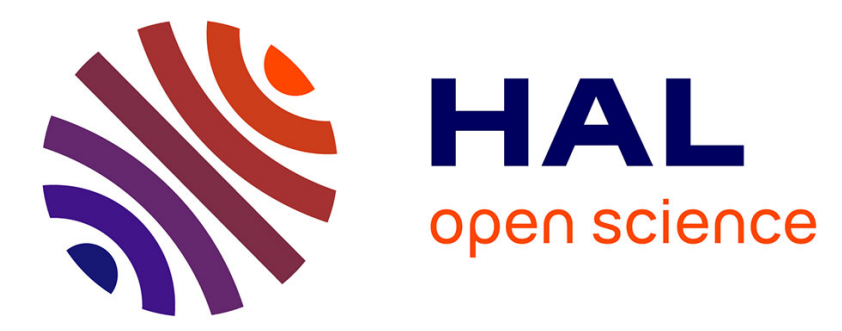

\title{
Thermal rectification in asymmetric two-phase nanowires
}

Paul Desmarchelier, Anne Tanguy, Konstantinos Termentzidis

\section{To cite this version:}

Paul Desmarchelier, Anne Tanguy, Konstantinos Termentzidis. Thermal rectification in asymmetric two-phase nanowires. Physical Review B, 2021, 103, pp.014202. 10.1103/physrevb.103.014202 . hal03331234

\section{HAL Id: hal-03331234 \\ https://hal.science/hal-03331234}

Submitted on 1 Sep 2021

HAL is a multi-disciplinary open access archive for the deposit and dissemination of scientific research documents, whether they are published or not. The documents may come from teaching and research institutions in France or abroad, or from public or private research centers.
L'archive ouverte pluridisciplinaire HAL, est destinée au dépôt et à la diffusion de documents scientifiques de niveau recherche, publiés ou non, émanant des établissements d'enseignement et de recherche français ou étrangers, des laboratoires publics ou privés. 


\title{
Thermal rectification in asymmetric two phases nanowires
}

\author{
Paul Desmarchelier \\ Universite de Lyon, CETHIL, INSA-Lyon, CNRS UMR5008, F-69621, Villeurbanne, France and \\ Universite de Lyon, LaMCoS, INSA-Lyon, CNRS UMR5259, F-69621, Villeurbanne, France \\ Anne Tanguy* \\ Universite de Lyon, LaMCoS, INSA-Lyon, CNRS UMR5259, F-69621, Villeurbanne, France and \\ ONERA, University Paris-Saclay, Chemin de la Hunire, BP 80100, 92123 Palaiseau, France \\ Konstantinos Termentzidis门 \\ Universite de Lyon, CETHIL, INSA-Lyon, CNRS UMR5008, F-69621, Villeurbanne, France
}

(Dated: January 13, 2021)

\begin{abstract}
Thermal rectification based on a new strategy of nanostructuration has been assessed with nonequilibrium molecular dynamics and wave-packet propagation simulations. The use of asymmetric crystalline-core/amorphous-conical-shell nanowires creates a direction-dependent thermal conductivity due to variable axial and radial phonon propagation/confinement. The origin of this effect is related to the combination of a crystalline/amorphous interface parallel to the heat flux, together with the variable amount of amorphous coating due to the conical shell of the nanowire. Physical insights of the rectification are given by the mean free path of phonons, the axial and radial energy transfer, the energy diffusivity, and the vibrational density of states restricted to different constitutive elements of the nanowire.
\end{abstract}

\section{INTRODUCTION}

The control of the energy carriers flow direction is fundamental for many applications. The first step toward this control was achieved for electronic flow, more than 100 years ago, with the invention of electronic diodes [1] and then transistors [2]. Rectification, or control over the flow direction, then has been observed for singlewavelength photons 3 and more recently for phonons [4]. The rectification of electron flux has allowed the development of electronics, but the processing of information is also possible with photons [5] or phonons [6]. Thermal diodes, or broad band rectification of heat carriers, were successfully built for electrons [7, phonons [8] and photons 9. These thermal diodes are useful among several applications in thermal management, as they allow both insulation from a hot environment, and heat dissipation for cooling processes.

Thermal rectification appears when the thermal conductivity $\kappa$ depends on the sign of the thermal gradient. Thus, it relies both on the space and temperature dependence of $\kappa$. Terraneo et al. 10] have shown that this multivariable dependence was possible using a monoatomic chain with an anharmonic interaction potential. This simple model has then been adjusted for different temperatures 11. Peyrard et al. have proposed a simple model estimating this rectification using the spatial and temperature dependence of $\kappa$ and optimized the rectification as a function of the temperature gradient [12]. The different strategies used to get thermal rectification are regrouped in a couple of recent review articles [13, 14].

\footnotetext{
* anne.tanguy@insa-lyon.fr

$\dagger$ konstantinos.termentzidis@insa-lyon.fr
}

Among them the main approaches are to join two materials with different thermal properties or to tailor the thermal properties through appropriate geometries at the nanoscale.

Thermal rectification can be induced by a proper interface between two materials. Within, this first strategy, several configurations have been proposed: In metal/insulator junctions the rectification is due to the direction dependent electron-phonon scattering rate at the interface [13. An insulator/insulator junction causes rectification as well if the thermal conductivity has different temperature dependence in each part [15]. Interface-induced rectification has been predicted between graphene nanoribbons with different carbon isotopes [16], or at the interface between a nanotube and a silicon substrate [17]. An interface-induced rectification has likewise been observed between two oxides of centimetric dimensions for a temperature difference of $30 \mathrm{~K}$ at temperatures below $100 \mathrm{~K}$ [18, 19]. Rectification can similarly be achieved with two semiconductors, due to the thermal boundary resistance at the interface [20], for example, in Si-Ge nanocomposites 21, 22. Finally, a crystalline $\mathrm{Si}$-amorphous polymer interface has been predicted to cause rectification due to the strong temperature dependence of the vibrational modes of the polymer causing the interfacial conductance to depend on the direction of the thermal gradient [23].

At the nanoscale, the geometry of the samples can induce rectification as well. Size effects impact the thermal conductivity and its dependence on the temperature [24] and can also promote localization of phonons [25]. A rectification obtained through an asymmetric nanostructure has been predicted for suspended graphene and diamond cones [26], for thin films [27, nanotubes [28], for graphene nanohorns [29], and for silicon nanocones [30]. 
Experimental studies have shown rectification induced by asymmetrically mass loaded nanotubes [31, asymmetric nanoribbons [32, and very recently, for silicon thin films with a gradual hole concentration [33. Thermal rectification has been predicted for nanowires (NWs) with specific geometries, either using telescopic shaped NWs 34 or NWs with sawtooth roughness [35. A gradual defect concentration, or a progressive alloying, have been proposed in simulation studies to induce rectification, as well [36. Finally, a gradient of interface density in a multilayer, accumulating geometrical and interfacial effects, has been shown to promote rectification [37.

In asymmetric geometries, where more mass or mass density is present on one side, the majority of the existing literature reports an increased heat flow toward the direction of decreasing mass or mass density. This bias direction has been linked to the increased scattering rate in the smaller part 32], the dependence of $\kappa$ on space and temperature [36, or the vibrational density of state (VDOS) mismatch between the small and large size [38. However, Ju et al. [39] have predicted a higher heat flux from the smaller to the larger crosssection of a Si trapezoid thin film, showing that the heat conduction is not always higher from the heavier regions to the lighter ones. Similarly, Wang et al. 32 have obtained a better flow from a defected graphene region to pristine one, and, more recently, Kasprzak et al. 33. have also showed a better heat flow from the high-porosity region toward the low-porosity region in a silicon thin film.

Crystalline/amorphous interfaces are known to perturb the phonon distribution, especially at the nanoscale [40]. These interfaces, when perpendicular to the flow, can even induce rectification, as has been shown for c-Si and a-PE interfaces [23]. Non perpendicular interfaces impact the flow as well. For instance, the interface between a-Si and c-Si is permeable to low-frequency phonons even at grazing angles 41 but represents a novel scattering source for vibrational waves, modifying the nature of low-energy vibrations and significantly anticipating the propagative to diffusive crossover 42 . Moreover, if phonons with frequency below $3 \mathrm{THz}$ are well transmitted, mode conversion allows the transmission of energy by higher-frequency phonons 43 . The free surfaces are also known to impact the phonon behavior in nanoobjects 44. The free surface effects seem to be crucial for the rectification through geometry. This is the case for graphene nanoribbons [32. Silicon as well is known to soften when its dimensions are reduced [45. Finally, ab initio simulations have shown a clear surface softening for Si NWs [46].

Focusing on nanowires allows studying simultaneously the reduction of dimensions and the role of free surfaces [4] or interfaces for the case of core/shell nanowires (CS-NW). An extensive study of surface effects in Si or Ge NWs has been made by Malhotra et al. [48. They have shown that, compared to the bulk, long wavelength phonons contribute less to the thermal conductivity. A core shell structure adds a supplementary interface, this time parallel to the heat flux, which leads to further decrease of the thermal conductivity. For this reason $\mathrm{Si}-\mathrm{Ge}$ CS-NW has been the subject of numerous studies showing that the interface between $\mathrm{Si}$ and Ge promotes localization of high-frequency phonons 49. In these CS-NW the difference of group velocities between core and shell induces couplings between longitudinal and transverse modes, giving rise to new coherent modes with eventually a localization of the longitudinal components [50]. The naturally occurring oxidized shells at the surface of Si NWs can induce similar effects. The amorphous shell is known to drastically reduce the thermal conductivity of the NWs. Early simulations reported a 100-fold decrease of $\kappa$ attributed to reduced phonon lifetime, in $\mathrm{Si}$ NWs with diameters below $4 \mathrm{~nm}$ 51. More recently, simulations have shown that dispersionless modes at the $\mathrm{Si}-\mathrm{SiO}_{2}$ interfaces might be responsible for the decrease of $\kappa$ [52]. A periodic modulation of the amorphous shell of a Si CS-NW has also been shown to reduce the thermal conductivity due to nanoconstrictions and phonon blocking [53].

A way to understand the rectification processes is to use atomistic modeling and study the effects of inverting heat flux direction. This can be addressed easily with the non nquilibrium nolecular nynamics (NEMD) method [38] where the link between temperature gradient and heat flow allows to derive the thermal conductivity. This can likewise be done with the approach-toequilibrium molecular dynamics 21] where the evolution of the temperature distribution from an initial gradient toward equilibrium allows estimating the thermal conductivity. With both methods, the inversion of heat flux direction is obtained with the inversion of heat reservoirs. Rectification can also be assessed for individual phonons thanks to wave-packet (WP) simulations [27, Monte Carlo for phonons [35], or atomistic Green's function [54].

Here we explore thermal rectification caused by a variable thickness of amorphous shells along the growth direction parallel to the heat flux. Our idea is to combine asymmetric shaped nanowires with an amorphous/crystalline interface which is parallel to the heat flux, with the objective to induce a positiondependent phononic dynamics in the crystalline core of the nanowire, due to the amorphous shell's thickness variation along the growth direction. In the second section the different used geometries are described along with the technical details of the study. Then we will show that it is possible to generate a rectification due to the enhancement of the thermal flux in the diameters increase direction. We finally, discuss the results in light of a detailed analysis combining the study of phononic dynamics with energetic (local kinetic energy) approaches and the factors leading to rectification. 


\section{SIMULATION METHODOLOGY AND CONFIGURATIONS}

\section{A. Studied configurations}

Several CS-NWs with or without variable thickness of shell are modeled using molecular dynamics (MD) simulations to study the induced rectification effect. The NWs are $50 \mathrm{~nm}$ or for a few cases $100 \mathrm{~nm}$ long and are composed of a cylindrical crystalline silicon core of constant diameter of $5 \mathrm{~nm}$, with an amorphous silicon shell of variable geometries. This amorphous shell can be seen as an approximation for the naturally occurring oxide shell [55]. The first NW has a constant shell thickness and it will be called cylindrical core shell nanowire and referred to as CL-CS-NW [see Fig. 1(a)]. The four other NWs with linearly increasing amorphous shell thickness resulting in conical-shaped NWs will be referred to as CO-CS-NWs [see Figs. 1(b)-(e)]. For the CL-CS-NW the added amorphous layer is $1 \mathrm{~nm}$ thick. For the CO-CSNWs the added shell layer goes from 0 at one extremity to $0.9,1.9,3.8$, or $7.6 \mathrm{~nm}$ at the opposite extremity. The four CO-CS-NWs configurations correspond to an opening angle of $\phi=\arctan \left(\frac{R_{\text {Shell }}-R_{\text {Core }}}{L}\right)$ of $1.1^{\circ}, 2.2^{\circ}, 4.4^{\circ}$ or $8.8^{\circ}$ respectively (see Fig. 2 ), with $R_{\text {Shell }}$ the maximal shell radius, $R_{\text {Core }}$ the core radius, and $L$ the NW length. The last geometry, a telescopic-shaped NW with a $3.5 \mathrm{~nm}$ amorphous thick shell covering half of its length, will be referred as TE-CS-NW [see Fig. 1(f)]. This shell thickness is chosen to have the same amorphous fraction as the CO-CS-NW-4.4 for the chosen NW length $(50 \mathrm{~nm})$. The dimensions, number of atoms, crystalline fraction, and effective radii the NWs are summarized in Table 1

The CS-NWs are created from the assembly of an amorphous shell and a crystalline core, with the method proposed by France-Lannord [55] and and similar to the one used in Ref. 42 to generate nanocomposites. First the shell, with a specific geometry for each configuration, is cut out of an a-Si bulk block. This a-Si sample is the same as the one used by Tlili et al. [42. The original sample is a cube with a side length of $17.4 \mathrm{~nm}$, which is repeated until it reaches the appropriate size before carving out the shell. The empty shells are then filled with a c-Si cylinder, with $\langle 100\rangle$ as the growth direction of the NWs. We apply free boundary conditions around the free surface, for this purpose we embedded the nanowire in a box of far larger dimensions in the perpendicular directions $(25 \mathrm{~nm})$, while periodic boundary conditions are imposed in the growth direction. A conjugated gradient energy minimization is performed to reach a local mechanical equilibrium. After that, the system is annealed at $100 \mathrm{~K}$ during $10 \mathrm{ps}$. Before this annealing, the box size is increased in the growth direction to prevent thermal stresses. The NW is then quenched using a conjugated gradient energy minimization to first remove the kinetic energy. A second energy minimisation is finally performed, at zero tensile stress inducing a small creep in the periodic direction (that is, strain evolution

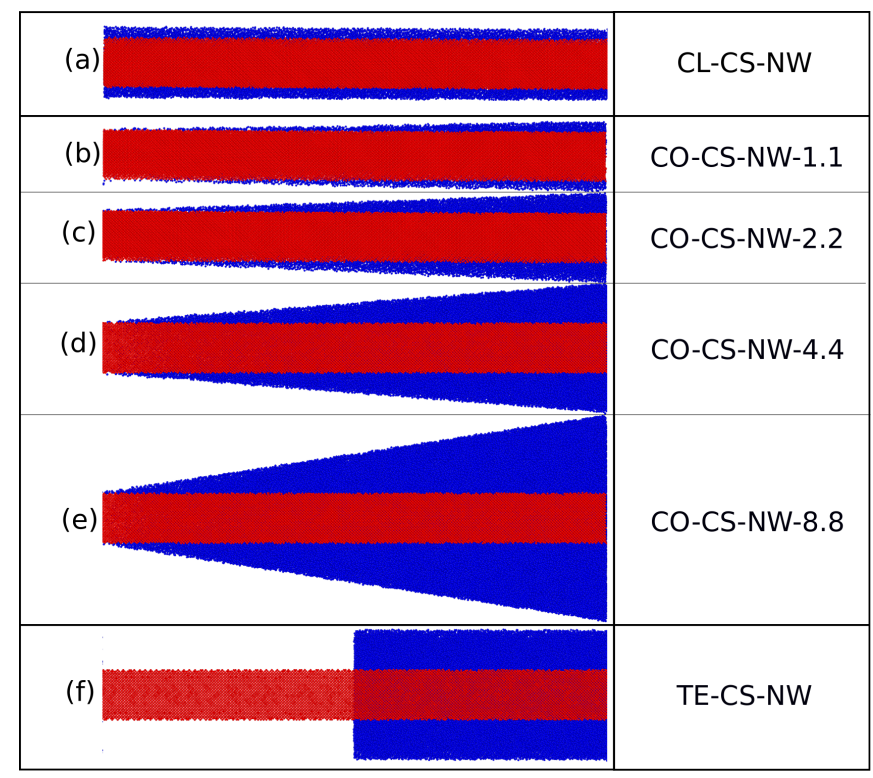

FIG. 1. Cross-sectional view along the growth direction of the CL-CS-NW (a), the CO-CS-NWs [(b)-(e)] with four opening angles of the amorphous shell, and, finally, the TE-CS-NW (f). Images are obtained from OVITO MD visualization software [56]. The red colored atoms correspond to the crystalline phase, while the blue ones to the amorphous phase.

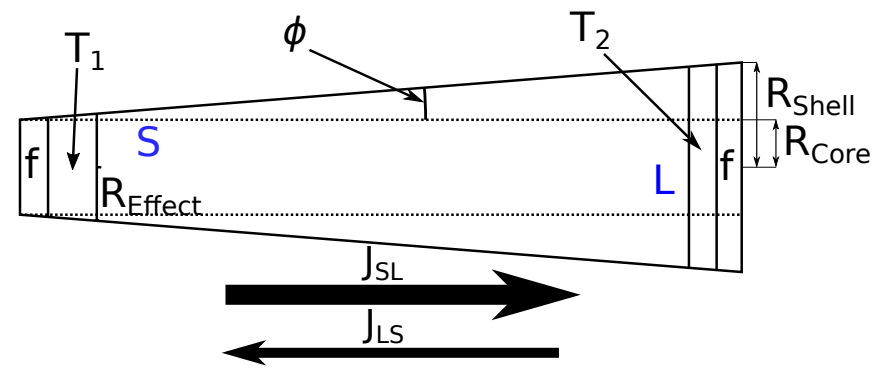

FIG. 2. Schematic representation of the NWs, with the thermostats $\left(T_{1}, T_{2}\right)$ and fixed atomic positions (f) on the conical CS configurations. Several characteristic elements, such as the effective, core, and shell radii, and the angle of the amorphous shell opening and large (L) and small (S) extremities of the NW are noted along with the flux direction $J_{S L}$ and $J_{L S}$.

at a global constant stress). To validate that the system is at equilibrium the fact that the spontaneous evolution of the system over 10 ps does not result in atomic kinetic energy above $10 \mathrm{feV}$ is checked. This minimization eliminated the thermal noise that could perturb the wave-packet simulation discussed later. These steps and all the MD simulations are performed using LAMMPS software [57]. We used a modified SW interatomic potential [58] allowing a more realistic modeling of the interface between c-Si and a-Si in terms of crystalline/amorphous interfacial energy and regular atomic energies inside the two phases [55. 


\begin{tabular}{|c|c|c|c|c|c|c|c|}
\hline & \multirow{2}{*}{ CL-CS } & \multicolumn{5}{|c|}{$\mathrm{CO}-\mathrm{CS}$} & \multirow{2}{*}{ TE-CS } \\
\hline & & $1.1^{\circ}$ & $2.2^{\circ}$ & & & $8.8^{\circ}$ & \\
\hline$R_{\text {Effect }}(\mathrm{nm})$ & 3.5 & 2.6 & 2.6 & 2.8 & 3.1 & 3.5 & 2.5 \\
\hline$R_{\text {Shell }}(\mathrm{nm})$ & 3.5 & 3.46 & 4.42 & 6.3 & 10.1 & 10.1 & 6.0 \\
\hline$L(\mathrm{~nm})$ & 50 & 50 & 50 & 50 & 100 & 50 & 50 \\
\hline$N_{t}$ & 95029 & 69617 & 95342 & 161353 & 689023 & 345766 & 161340 \\
\hline$N_{c} / N_{t}$ & $51 \%$ & $70 \%$ & $51 \%$ & $30 \%$ & $14 \%$ & $14 \%$ & $30 \%$ \\
\hline
\end{tabular}

TABLE I. Effective radius, shell radius, length, total number of atoms and fraction of atoms belonging to crystalline phase for the 3 groups (in total 7 configurations) of NWs.

\section{B. Non Equilibrium Molecular Dynamic}

The effective thermal conductivity of all configurations of NWs are evaluated using the NEMD method. A temperature gradient is imposed across the extremities of the NW and the thermal conductivity is then derived from $\kappa=\frac{J \Delta x}{S_{E f f e c t} \Delta T}$ with $J$ the energy transfer rate between the thermostats, $S_{E f f e c t}$ the effective section, and $\frac{\Delta T}{\Delta x}$ the temperature gradient. Before setting up the temperature gradient we equilibrated the NW at $300 \mathrm{~K}$ for 500 ps with an NPT (isothermal-isobaric) thermostats, to prevent buckling. After this thermalization, the boundary conditions in the growth direction are switched from periodic to fixed to avoid a direct interaction between the heat baths. Additionally, the position of the atoms within $1 \mathrm{~nm}$ of the simulation box edges are fixed. Finally, the two thermostats are added next to the fixed atoms (see Fig. 2.)

The number of atoms in the thermostats on the large side and the small side is kept similar within $1 \%$ by varying their thicknesses between 0.5 and $5 \mathrm{~nm}$. A simple velocity rescaling is used to maintain the cold side at $280 \mathrm{~K}$ and the hot side at $320 \mathrm{~K}$, or $260 \mathrm{~K}$ and $340 \mathrm{~K}$ respectively, for simulations with a $\Delta T$ of $80 \mathrm{~K}$. The time step of the Verlet scheme integration is $5.0 \times 10^{-4} \mathrm{ps}$. The stability of the temperature profile obtained after the first 1000 ps indicates that a steady state is reached, the flux is then computed over the next 1000 ps. For the 100-nm-long-NW theses times are doubled (for more details, see Appendix A. The considered flux is the mean value of five independent simulations with different initial velocity distribution to decrease the statistical error (see Fig. 11 in the Appendix A). The heat flux is computed twice, switching the hot and cold thermostats to obtain the heat flux in both directions. When the hot thermostat is situated in the smaller section, we call it SL flux (small to large) and in the opposite direction LS flux (see Fig. 2). The effective section $S_{\text {Effect }}$ considered for the computation of $\kappa$, if not otherwise specified, is the limiting cross section for heat transport, that is, the smallest section at the end of the thermostat $T_{1}$ in Fig. 2 (the effective radius $R_{\text {Effect }}$ is reported in Table I). The temperature gradient is defined as the temperature difference between the thermostats divided by the distance separating the two thermostats $\Delta x \approx L$.
The thermal rectification is computed as

$$
\eta=\frac{\left\langle\kappa_{S L}\right\rangle-\left\langle\kappa_{L S}\right\rangle}{\left\langle\kappa_{S L}\right\rangle}
$$

with $\langle\kappa\rangle$ the mean thermal conductivity over the repetitions. $\kappa$ can be replaced by $J$ in the previous equation as the thermal gradient is the same except for the sign and the effective section is the same. The uncertainty is derived from the standard deviation $\sigma$ of the thermal conductivity value:

$$
\Delta \eta=\eta\left(\frac{\sigma\left(\kappa_{S L}\right)}{\left\langle\kappa_{S L}\right\rangle}+\frac{\sigma\left(\kappa_{L S}\right)}{\left\langle\kappa_{L S}\right\rangle}\right)
$$

\section{Wavepacket propagation through the NWs}

In order to get physical insights from the phononic nature of the phenomenon, a wave-packet method similar to the one described by Beltukov et al. [59] is used. The wave packet is generated through a Gaussian windowed sinusoidal force impulsion,

$$
f=A \sin [2 \pi \nu(t-3 \tau)] \exp \left[-\frac{(t-3 \tau)^{2}}{\left(2 \tau^{2}\right)}\right],
$$

imposed to the atoms in a 0.2 -nm-thick central slice. The amplitude $A$ is chosen small enough to avoid anharmonic effects, and the resulting displacement is of maximum $0.15 \times 10^{-4} \mathrm{~nm}$. The spreading of the Gaussian window $\tau$ is chosen to be sufficiently small to offer a compromise between spatial extension of the WP compared to the NW length and the resolution in the frequency space. The used value is $0.36 \mathrm{ps}$. The spectral resolution can be defined as the width of the Fourier transform of the window, $\delta \nu \approx 1 / 2 \pi \tau \approx 0.4 \mathrm{THz}$. As the excitation frequency $\nu$ will be between 1 and $15 \mathrm{THz}$, the spectral spreading is of the order of the frequency for the lowestfrequency value. The force $f$ is applied either in the growth direction, creating longitudinal (L) waves or perpendicular to the growth direction, creating transverse (T) waves. Alternatively the force can be applied in a random direction, preventing a coherent wave and thus keeping only the diffusive part of the motion [60]. After the excitation the WP is recorded every $0.01 \mathrm{ps}$ from the creation of the impulsion until the wave reaches the 
periodic boundaries. The local kinetic energy per $\delta x$ wide slice is recorded along the whole NW and also the core and the shell separately. For the visualization of the kinetic energy distribution along the $\mathrm{NW}$ we used a Savitzky-Golay 61] second-order polynomial filter with a window of $7 \mathrm{~nm}$. Additionally, the position and kinetic energy of every atom is recorded every $0.3 \mathrm{ps}$ in order to get the full picture of the energy distribution.

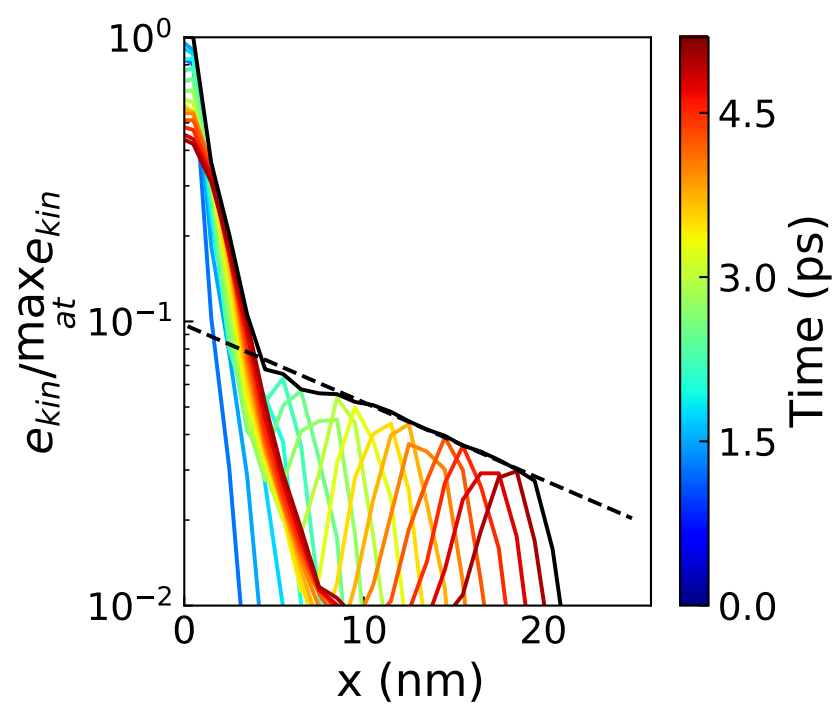

FIG. 3. The envelope is defined as the maximal amplitude of the wave at a given position (black solid line). The energy distribution for some time steps are represented with different colors. The dashed line corresponds to the fit used to determine the MFP. Here the reference (0) position is the position where the excitation is created, and the initial time corresponds to the Eq. (3).

The mean free path (MFP) of the phonon is estimated from the decrease rate of the maximum amplitude of the nonrandom wave packets (envelope) as a function of the distance from the excitation source (see Fig. 3). The envelope of a wave packet traveling ballistically follows a Beer-Lambert law (exponential decrease) [59]. As the excitation is made at the center of the NW the MFP, it can be evaluated in both directions, small to large (SL) and large to small (LS), with the same measurement.

Even for the non random excitation, we have both a diffusive and a propagative contribution to the energy transfer. The diffusive part is shown as a spatial peak in kinetic energy that broadens slowly and the propagative part is a Gaussian shaped lobe that is translated as the WP propagates (see Fig. 3). In order to obtain a meaningful MFP, the decrease must be evaluated on the propagative part only. To this end, the decrease rate of the envelope is evaluated from $10 \mathrm{~nm}$ away from the initial excitation to $4 \mathrm{~nm}$ away from the simulation cell boundary (see Fig. 33). This choice allows avoiding artifacts due to the diffusive part. The corresponding decrease rate is evaluated through a least-squares fit to an exponential decay. Finally, as mentioned in Sec. IIA, before the ex- citation, there is no initial velocities in the system. This is necessary to get rid of any thermal noise in the energy distribution. Thus, the computed MFP with our method here does not take into account temperature effects.

The energy diffusivity is computed with the same method as in Beltukov et al. 60]. The average square diffusion front after a random direction excitation for each given frequencies is computed:

$$
R^{2}(t)=\frac{1}{E_{t o t}} \sum_{i=0}^{N} x_{i}^{2} E_{i},
$$

with $N$ the number of slices, $i$ the slice index of width $\delta x, x_{i}$ the distance to the excitation, and $E_{i}$ the kinetic energy of the $i$ th slice. The diffusivity is given by the time dependence of $R^{2}$ thanks to the equation of onedimensional diffusion,

$$
R^{2}(t)=2 D(\nu) t
$$

In each case $D(\nu)$ is computed through a least-squares fit. In order to compute the diffusivity for each direction, the half of the NW, either LS or SL, is considered.

\section{Vibrational density of state}

The VDOS is evaluated through the Fourier transform of the velocity auto correlation function (VACF) [62. To this end, the system is thermalized at $50 \mathrm{~K}$ for $100 \mathrm{ps}$ with a Nosé-Hoover thermostat. The VACF averaged over the chosen particles is then recorded over the next 100 ps without thermostating. The Fourier transform is finally filtered using a Savitzky-Golay polynomial filter 61]. With this method, we evaluated the (partial) VDOS as a function of the distance to the center of the NWs and for different segments of the NW in the $x$ direction.

\section{ENERGY FLUX AND RECTIFICATION}

We will now observe and discuss the effect of the asymmetry of amorphous shells on the thermal conductivity of nanowires. To do so, we will focus first on the heat flux direction. In Fig. 4 both the heat flux or the thermal conductivity and the rectification (green right axis) for different directions ( $\mathrm{S}$ to $\mathrm{L}$ and $\mathrm{L}$ to $\mathrm{S}$ ), as a function of the opening angles of the samples, or as a function of the amorphous fraction for the given NW length are depicted. It can be seen that the flux and the thermal conductivity in the SL (red empty circle) direction is superior to the heat flux or thermal conductivity in the LS direction (blue star). There are two ways to define rectification; using the heat flux or the thermal conductivity. In symmetric structures the two quantities will give the same rectification. In our case, as the studied configurations are asymmetric, we have chosen the heat 
flux because it is independent of the choice of the cross section [in Fig. 4(a) and 4(b)]. Nevertheless, we have also depicted the results in terms of the effective thermal conductivity with two different cross sections in Fig. 4 (c) using the smallest cross section, related to the vision of the nanoconstriction which usually drives the phenomena at the nanoscale, and in Fig. 4(d) using the cross section next to the hot thermostat related to the vision of the energy dissipation area for a given system. We discuss later, the pertinence of the choice between the two cross sections.

In Figures 4(a) and 4(c), it appears that the asymmetric amorphous shell induces a thermal rectification for the CO-CS-NWs with the two largest opening angles $\left(4.4^{\circ}\right.$ and $\left.8.8^{\circ}\right)$ and for the TE-CS-NW. The rectification, measured on the heat flux $(\eta=5 \%)$, is of the order of the rectification obtained experimentally for a similar system 31. However, the CO-CS-NW-8.8 has a slightly lower $\eta$ than CO-CS-NW-4.4, showing that the relation between the opening angle and the rectification is not monotonic. Moreover, our simulations indicate that there is a threshold effect for rectification in either opening angle between $2.2^{\circ}$ and $4.4^{\circ}$ or in amorphous fraction here, between $50 \%$ and $70 \%$ for the length $L=50 \mathrm{~nm}$. The rectification measured for the CO-CS-NW-1.1 and CO-CS-NW-2.2 are not significant. Thus, an important thickness of amorphous shell is necessary to modify the direction of the heat flux in the asymmetric NWs and thus induce rectification. Moreover, as the telescopic geometry induces less rectification than the CO-CS-NW-4.4 with similar amorphous fraction we can conclude that the asymmetry of the amorphous shell plays an important role, but is not the only factor responsible for the observed rectification.

Interestingly, the total heat flux increases monotonously with the amorphous fraction [Fig. 4 (a) and (b)]. As the crystalline part has a constant diameter, an increasing amorphous fraction means that the section through which the heat flux can go through increases. The core diameter being constant, a significant part of the heat flux has to go through the amorphous shell. However, the thermal conductivity of a-Si being roughly two orders of magnitude below the c-Si one, a smaller enhancement was expected. This important difference in the total flux between the CO-CS-NWs indicates a considerable energy exchange at the interface between the core and shell.

All the CO-CS-NWs share similar thermal conductivity values. Once normalized by the cross section, here the smallest section between the thermostat (see TableI), the disparities in flux disappear [see Fig. 4 (c)]. It is noteworthy that the TE-CS-NW has much larger thermal conductivity due to the reduced effective section; similarly, the thermal conductivity of CL-CS-NW, having a large effective cross section, is lower. With this effective section definition, neither the effective cross section nor the distance between the thermostats varies, thus the rectification is independent of the choice of $J$ or $\kappa$. However, the thermal rectification can change with another choice for the effective cross section. For instance, in Fig. 4(d), we have used for the estimation of the thermal conductivity the section which corresponds each time to the hot thermostat and thus the section of the incoming flux. This choice of effective section results in very high $\eta$ values $(90 \%)$. However, this variation is mainly due to the change of the effective section depending on the direction the thermal gradient. To compare the direction of propagation a comparison of the flux or of the effective thermal conductivity using always the smaller section seems more useful. In all cases, the thermal conductivity estimations are of the same order as in the previous simulation of Verdier et al. 63. However, a more precise comparison between our thermal conductivity and previous results is not possible due to potential size effects 64].

To get an idea of the size effect on the rectification coefficient, we have studied longer NW. The results obtained for the 50- and 100-nm-long CO-CS-NW-4.4 are displayed in Table II. For the longer NW two temperature differences between the thermostats are tested: one with the usual $\Delta T$ of $40 \mathrm{~K}$ and one with a $\Delta T$ of $80 \mathrm{~K}$ to keep the same temperature gradient, $\Delta T / L$, as for the $50-\mathrm{nm}$ NWs. Due to the computational cost for the 100-nm NW only two independent runs were done. First, one can notice that the rectification still appears when the length is doubled and that it can even increase for the $\Delta T$ of $40 \mathrm{~K}$. However, in increasing the $\Delta T$ to $80 \mathrm{~K}$, the rectification decreases. These results show that there is not a systematic dependence of the rectification on the $\Delta T$, or on the length of the asymmetric NW and that the observed phenomena are too complex to be expressed by a simple equation of rectification vs. $\Delta T$ or length. Due to computational CPU restrictions, we could not go further in the investigation of the impact of these two parameters on the thermal rectification. The lower $\Delta T / L$ and increased length seems to favor the rectification.

Finally, it is interesting to note here that the main direction of the heat flux is opposite to the direction obtained for homogeneous samples with conical shape as shown by Wang et al. [26. We will discuss this point later. For a more comprehensive study, we will first relate the heat flux to the density of eigenmodes supporting it.

\section{VIBRATIONAL PROPERTIES}

The effects of the geometry on the phonon heat transport can be evaluated qualitatively by analyzing the contribution of individual phonons. The mean free path is a way to quantify the effect of the geometry on the ballistic part of the propagation for individual phonons. For example, the rectification could be related to a higher MFP in a specific direction. This is assessed in Fig. 6 where the MFP of longitudinal and transverse phonons with frequencies between 1 and $15 \mathrm{THz}$ in both directions of propagation in the NW (LS and SL) are depicted for the 

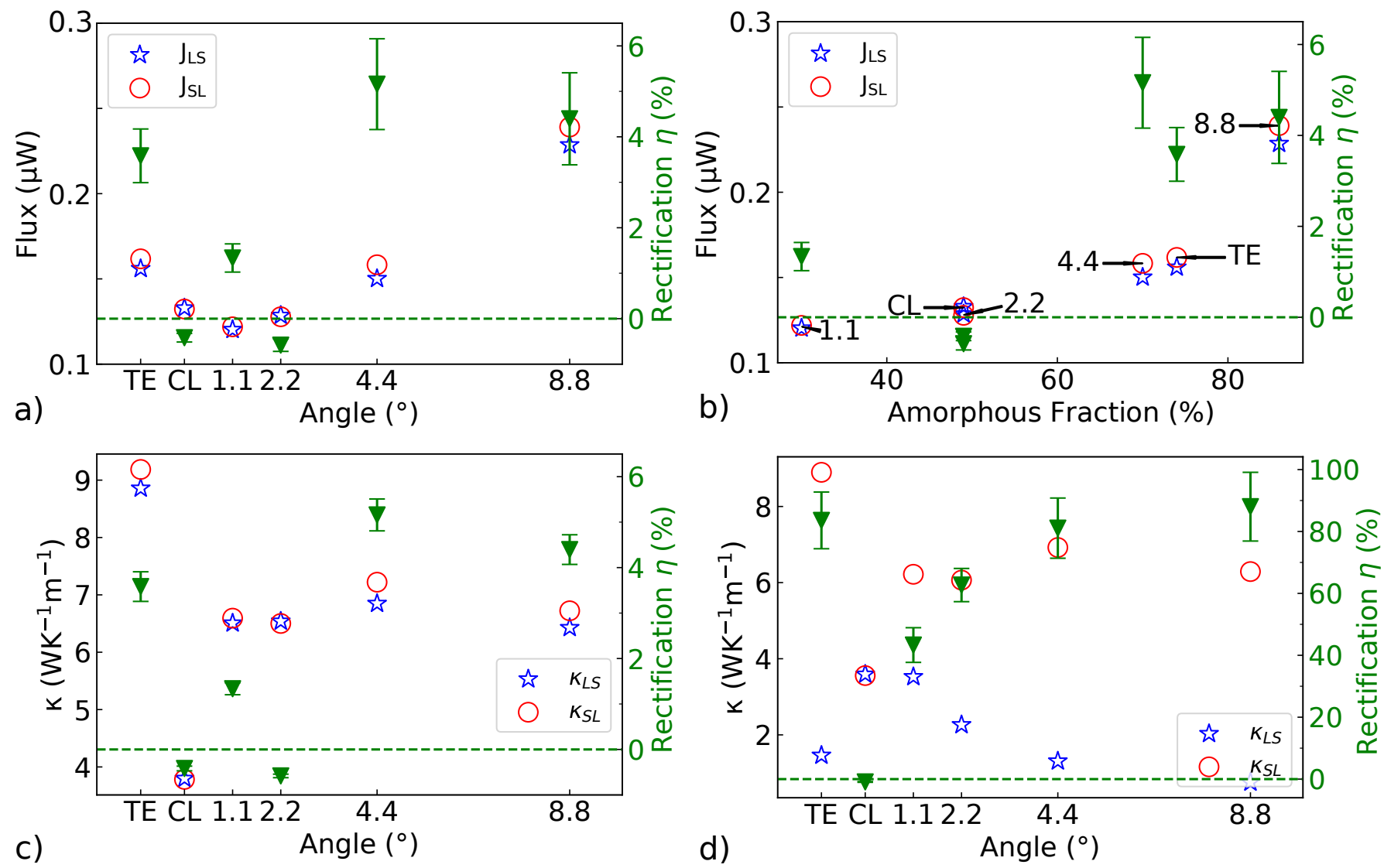

FIG. 4. Power exchanged and rectification for all the 50-nm-long NWs as a function (a) of the opening angle of the amorphous shell (b) of amorphous fraction or thermal conductivity and rectification as a function (c) of the opening angle computed with the effective section defined in Table $\mathrm{I}$ or $(\mathrm{d})$ with the section next to the hot thermostat. The different configuration are identified by their opening angles for the CO-CS-NW and by TE or CL for the TE-CS-NW and the CL-CS-NW. On the left $y$ axis, $\kappa_{L S}$ or $J_{L S}$ are represented by blue stars and $\kappa_{S L}$ or $J_{S L}$ by red circles. The rectification values on the right $y$ axis are represented by green triangles, with error bars determined from Eq. 2) and the green dashed line gives the zero rectification.

\begin{tabular}{lcccccc}
\hline \hline $\mathrm{L}(\mathrm{nm})$ & $\Delta T(\mathrm{~K})$ & $\Delta T / L\left(\mathrm{~K} \mathrm{~nm}^{-1}\right)$ & $J_{S L}(\mu \mathrm{W})$ & $J_{L S}(\mu \mathrm{W})$ & $\eta(\%)$ & $\#_{\text {run }}$ \\
\hline \multirow{5}{*}{50} & 40 & 0.8 & 0.158 & 0.150 & 5.2 & 5 \\
\hline \multirow{2}{*}{100} & 40 & 0.4 & 0.126 & 0.115 & 8.4 & 2 \\
\cline { 2 - 7 } & 80 & 0.8 & 0.246 & 0.238 & 3.2 & 2 \\
\hline \hline
\end{tabular}

TABLE II. Size and temperature impact on thermal rectification: length of NW, imposed $\Delta T$ between the two thermostats, temperature gradient, mean flux in the two directions, rectification, and number of independent runs for the CO-CS-NW-4.4.

CO-CS-NWs and the CL-CS-NW, along with the VDOS of the configuration. Due to the free surface perpendicular to the propagation direction next to the excitation source the MFP of TE-CS-NW cannot be reliably studied with the method described in Sec. IIC.

For longitudinal phonons the MFP is higher in SL than in LS direction at low frequencies $(1-3 \mathrm{THz})$ for CO-CSNW-4.4 and CO-CS-NW-8.8. The transverse phonons at $1-2 \mathrm{THz}$ have, similarly, a higher MFP in the SL direction. As expected, this characterizes an asymmetric transport favoring the SL direction. However, this is not the case for all frequency ranges. For example, the MFP in the LS direction is higher in the frequency range of $3-5 \mathrm{THz}$ for longitudinal phonons and in the $1-6 \mathrm{THz}$ frequency range for transverse phonons for CO-CS-NW1.1. If the MFP at low frequency controlled the rectification behavior, then the rectification would be negative for CO-CS-NW-1.1 and positive for CO-CS-NW-8.8 and CO-CS-NW-4.4 (in Fig. 6). However, no significant rectification is measured for the CO-CS-NW-1.1. Thus, the MFP of low-frequency phonons, may contribute to rectification, but cannot explain it alone.

Note that the frequency dependence of the MFPs in all samples is somehow unusual, especially the very high peak at high frequencies $(11 \mathrm{THz})$. In bulk c-Si 65] and a-Si 66, or for larger NWs [67, such a high peak has not been observed. A smaller peak in the longitudinal MFP was already related before to the attenuation of 


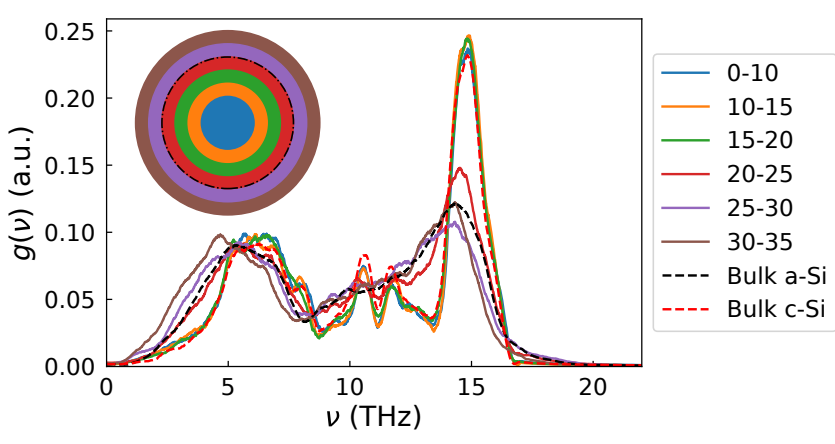

FIG. 5. Vibrational density of state as a function of coaxial (hollow) cylinders of thickness of $0.5 \mathrm{~nm}$ for the CL-CS-NW, compared with the bulk VDOS of a-Si and c-Si in dashed lines. The inset links the colors and the considered cylinders, the dot-dashed line represent the crystalline amorphous interface.

transverse waves in a-Si and to the resulting lack of interactions between longitudinal and transverse waves [59]. Moreover, in the CO-CS-NWs, the density of modes between 8 and $12 \mathrm{THz}$ is even smaller than in a-Si, as can be seen in the third row of the Fig. 6] inducing less dispersion of the WP, and thus a subsequent increase of the MFP in this frequency range. This increase can also be linked to the fact that only the core seems to participate to the ballistic heat transfer at these frequencies (see Fig. 8). A rapid decrease of the MFP between 0 and $4 \mathrm{THz}$ followed by a plateau was nevertheless predicted for square NW section of similar cross-sectional area [68. The lack of second maximum in the MFP at high frequency in the previous study might be due to the temperature effect, as in our case the MFP is computed without temperature.

As the studied systems are composed of a large amorphous part, it is important to consider the influence of the nonpropagative modes to complete the study. To this end, the energy diffusivity for the different frequencies is evaluated in the SL and LS directions. The results are shown in the Fig. 6 (third row). First, one can observe that the diffusivity in our systems is comparable to the bulk amorphous silicon diffusivity [59]. This similarity is notable even for the CO-CS-NW-1.1 where the amorphous shell is very thin, especially in the LS direction. Moreover, a distinct peak is visible around $8 \mathrm{THz}$ for all configurations. This peak was previously observed for amorphous silicon and was linked to the Ioffe-Regel criterion for transverse phonons [59. In increasing the overall amorphous fraction ( $1.1^{\circ}$ to $8.8^{\circ}$ opening angles) of the systems, the diffusivity decreases. Nevertheless, when comparing the directions for the larger opening angle $4.4^{\circ}$ and $8.8^{\circ}$, it appears that the diffusivity is higher in the SL direction where the amorphous shell becomes thicker. This higher diffusivity suggests a better diffusive transport of heat in the same direction as for the global heat flux. Thus at first glance, the diffusive transport seems to participate to the rectification, even if it is not simply related to the amorphous fraction evolution.
Let us now look at the global vibrational density of states. The VDOS of the core of the CO-CS-NW-4.4 is not affected by the position along the growth direction but only by the distance from the interface with the amorphous shell (see Fig. 7). The VDOS in the core is not affected by the thickness of the amorphous layer above it. The rectification can thus not be caused by altered vibrational properties of the core itself but only by a dynamical core shell interaction. Moreover, the VDOS of the CL-CS-NW (Fig. 5) shows a redshift in the outer part of the shell implying that more energy can be carried by low frequencies at the outer surface. Assuming that the behavior is similar in the CO-CS-NWs, the increase of the outer surface available in the SL direction allows carrying more energy than in the opposite direction. The transport at the surface can thus be an additional reason of the observed rectification.

The importance of the surface transport can also be assessed in Fig. 8 by looking at the kinetic energy per atom along the width of the NW, and at different times. At $2 \mathrm{THz}$ there is a clear wave propagation at the surface, for the CL-CS-NW as well as for the CO-CS-NW-8.8. Moreover, a noticeable asymmetry appears for the COCS-NW-8.8. In the LS direction the energy concentration is very inhomogeneous: It is far higher in the core and at the free surface (identified by the decreased atom density) than in the SL direction. The comparison between CL-CS-NW and CO-CS-NW-8.8 shows that the geometrical asymmetry of the shell changes the distribution of energy in the shell and core. The distribution profile of the CL-CS-NW is very similar to the distribution profile of the CO-CS-NW-8.8 in the LS direction. This similarity hints that the proximity of the free surface has a strong influence on low-frequency phonons. At $12 \mathrm{THz}$ theses differences disappear; the energy mainly flows in the core for both directions.

Moreover, for low frequencies there is a net energy exchange between the core and the shell. This is visible through the unbalanced energy ratio between the two sides of the impulsion in Fig. 9 in the core and shell. There is more energy in the shell and less in the core for the SL part of the NW for all CO-CS-NWs. As the energy is overall evenly distributed between the SL and LS parts when considering the whole NWs, this distribution is only possible through an energy transfer between the two phases. The shell transfers energy back to the core in the LS direction, especially in the low-frequency range. This transfer is most likely a leading factor for the rectification. The energy distribution for the shell of COCS-NW-1.1 seems different from the other CO-CS-NW. This can be explained by the reduced number of atoms in the shell [see Fig. 11(b)] making the energy ratio more sensitive to small variation. 


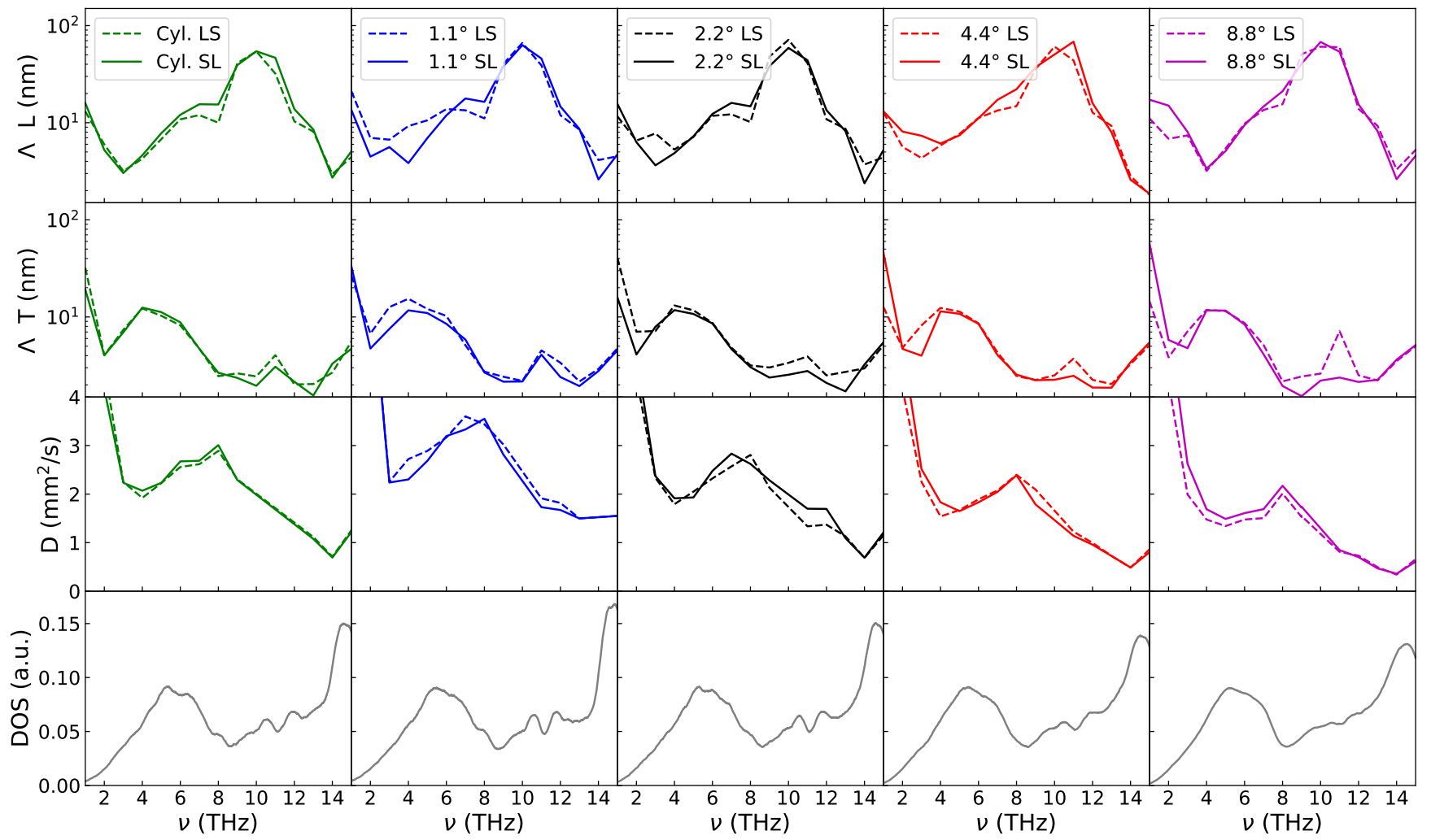

FIG. 6. Longitudinal (first row) and transverse (second row) MFP, diffusivity (third row), and VDOS (last row) for all studied configurations. The solid colored lines represent the SL direction and the dashed ones the LS direction. The different configuration are identified by their opening angles for the CO-CS-NW and by CL for the CL-CS-NW.

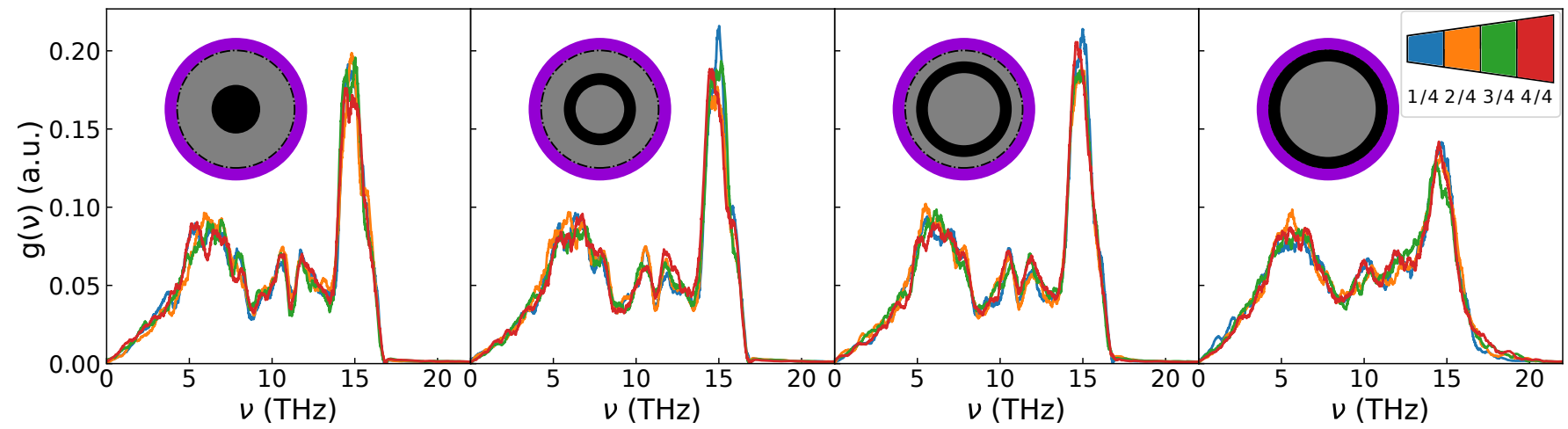

FIG. 7. Vibrational density of state as a function of the atoms belonging to a cylindrical ring of the crystalline part (four columns) and for different position along the CO-CS-NW-4.4 (colored lines). The insets link the column and the considered radius ranges. The black circle in the inset represent the part of the nanowire core considered, the gray the whole core, and the outer shell is represented by the purple outer circle and is not to scale. The mixed line represents the crystalline amorphous interface.

\section{KINETIC ENERGY VISION}

The temporal and spatial evolution of the wave packet can be analyzed qualitatively through the representation of the kinetic energy distribution along the NW (Fig. 10). After the initial nonrandom impulsion two parts are visible at all frequencies ranges for both geometries: a prop- agative wave traveling along the NW mainly inside the core of the NW and a slowly broadening diffusive peak. In Fig. 10 the propagative part manifests as distinct lobes that are shifted in the propagation direction at every time step. These features are particularly visible for the COCS-NW at $12 \mathrm{THz}$ (Fig. 10 first figure bottom row) but are present to some extent in all CS-NWs at all frequen- 


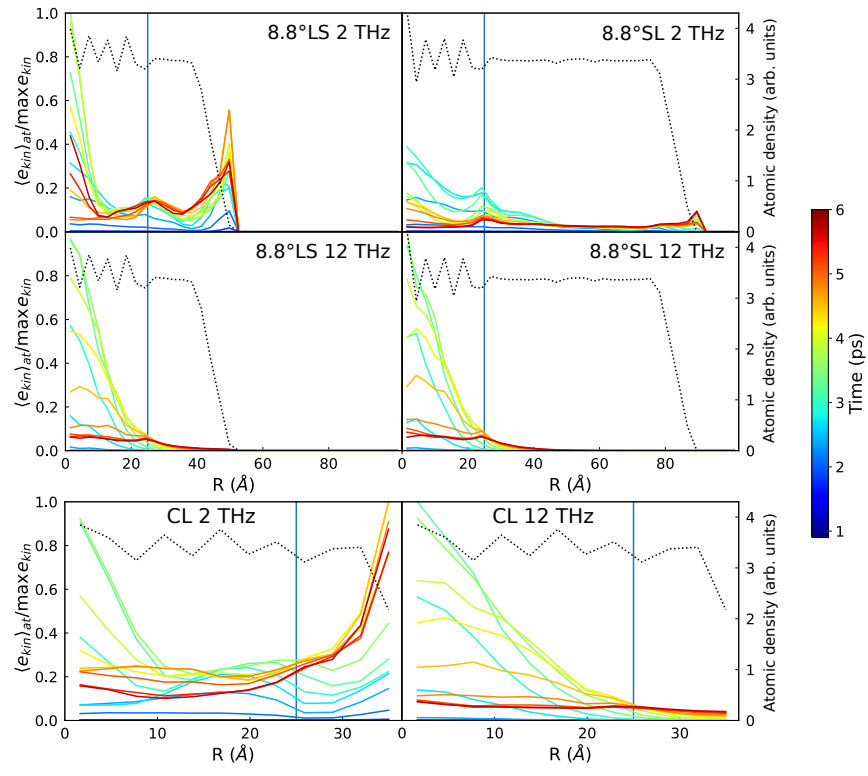

FIG. 8. Evolution of the mean kinetic energy per atom as function of the radius in 7 -nm-thick slice, $9 \mathrm{~nm}$ away from the excitation source after a longitudinal impulsion at different times, for the CO-CS-NW-8.8 (upper row) and the CL-CSNW (lower row). The energy values are normalized by the maximum value for at each given frequency. The black dotted lines represent the density of atoms in the slice and the vertical blue line represents the amorphous/crystalline interface.

cies below $15 \mathrm{THz}$. The diffusive peak is present in both shell and core. A diffusive peak was expected in the shell, as for most frequencies between 2 and $12 \mathrm{THz}$ the diffusive regimes can be observed after an excitation in a$\mathrm{Si}$ [59]. But its presence in the crystalline core was unexpected. This central peak is mostly visible at $12 \mathrm{THz}$ for the longitudinal excitation, and at $4 \mathrm{THz}$ for the transverse excitation, but is nevertheless already present at $2 \mathrm{THz}$ for both polarizations. This diffusive behavior of thin CS Si NWs was already observed by Donadio et al. [51, although this behavior was thought to be limited to the amorphous layer. As a purely propagative behavior is expected in c-Si, a diffusive part indicates either an energy transfer from the shell to the core or an influence of the interface on the propagation. The influence of the shell on the core vibrational properties has already been assessed in Fig. 5. Atoms in the core, within $0.5 \mathrm{~nm}$ from the interface have a very similar VDOS to the one of a-Si, but this limited effect is not sufficient to explain the diffusive transfer inside the whole crystalline core. This must be due to a strong dynamical effect induced by the long-range influence of the amorphous/crystalline interface.

We will focus now on the asymmetric behavior of the energy propagation in the CO-CS-NW-8.8 especially at low frequencies. The bias in transport favoring the SL direction at $2 \mathrm{THz}$ is confirmed by the ballistic transport clearly visible in one direction only, as can be seen in the first column of Fig. 10 representing the energy dis-

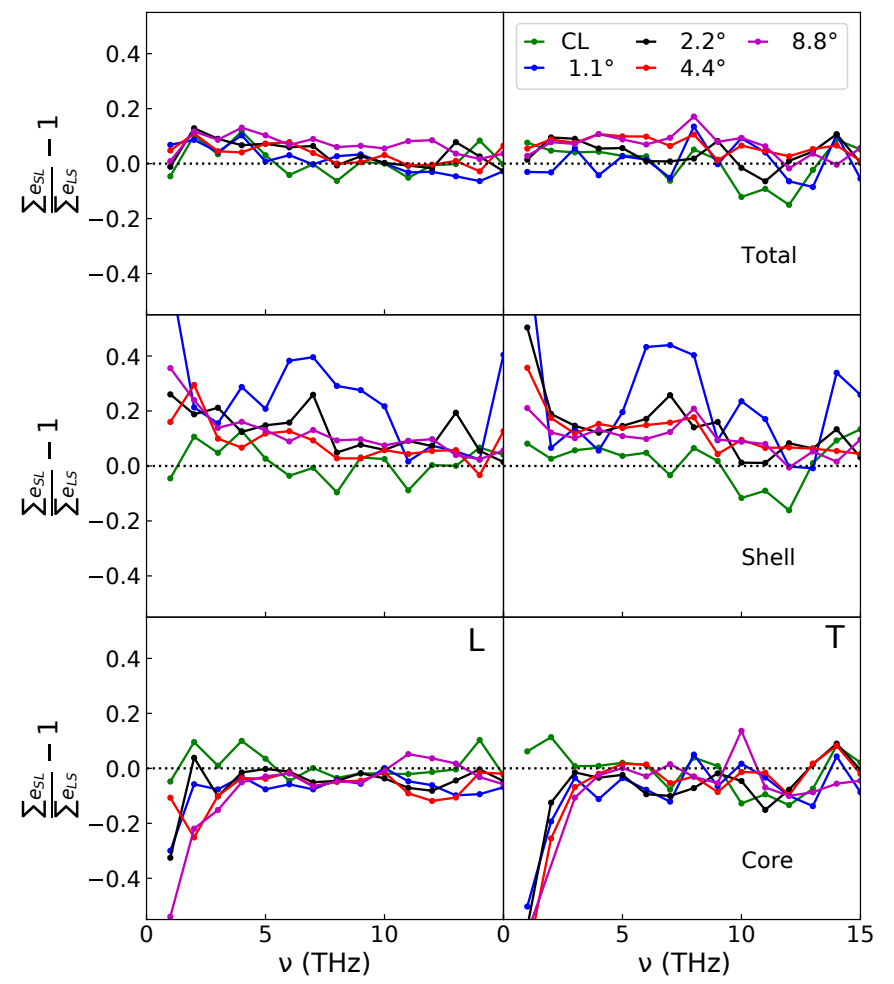

FIG. 9. Kinetic energy ratio: Sum of atomic kinetic energy in the SL direction ( $e_{S L}$, right of the impulsion) divided by the sum of atomic kinetic energy in the LS direction $\left(e_{L S}\right.$, left of the impulsion), and the mean from the end of the impulsion of propagation until the wave front reaches the boundaries. For the longitudinal (left column) and transverse (right column) excitation

tribution in the whole NW. This behavior also appears in the energy distribution in the longitudinal cross section in the central column of Table VI. In the SL direction the wave packet travels without coherence losses (the maxima and minima of kinetic energy are visible during the whole propagation), while in the LS direction the WP is more dispersed/attenuated. This dispersion seems to results from the interactions between the waves mainly propagating in the shell and that propagating in the core. The shape of the shell induces strong interactions between the waves supported by the shell and by the core, in the converging LS direction but keep them apart in the diverging SL direction. This interference is confirmed by the analysis of the displacement in the left column of Table VI In the SL direction the wave front travels through the NW without coherence loss, through core and shell alike. But in the LS direction the wave in the shell is phase shifted, with different displacement directions for a given $x$. This phase shift seems to interfere with the propagation in the core. This phenomenon is affected by the shells thickness: For a thicker shell the wave is less affected by the interface despite the structural changes (see Table VI), whereas for thinner shells, either for the CO-CS-NW-8.8 in the LS direction or for the CL-CS-NW, a clear phase shift occurs at the inter- 

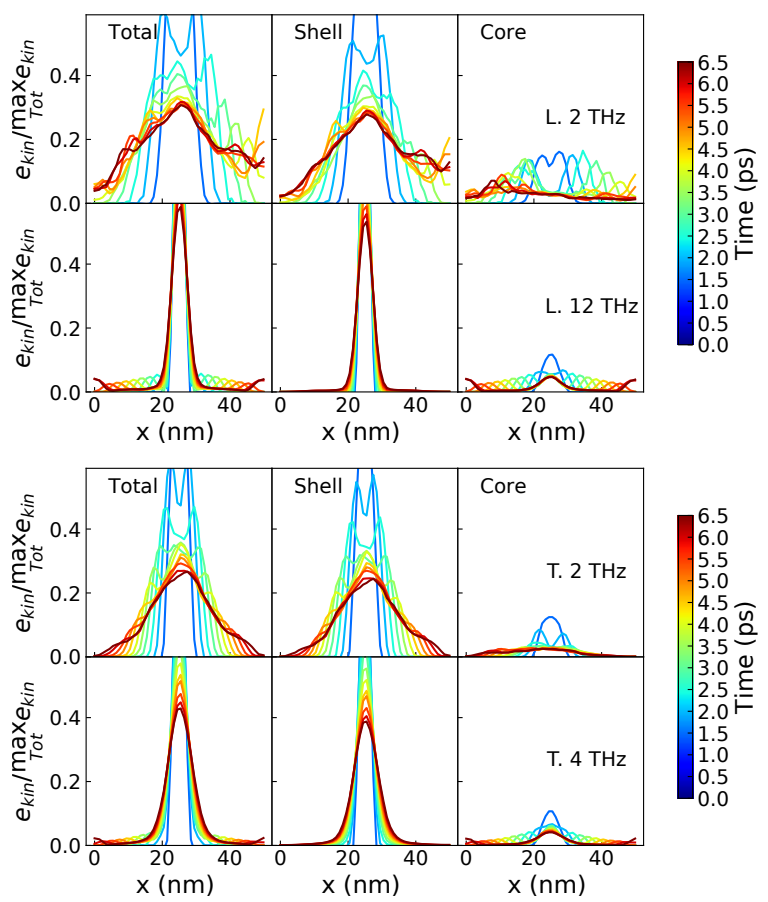

FIG. 10. Evolution of the kinetic energy distribution along the wire (sum of atomic kinetic energy in each individual slices filtered with a polynomial filter) after a longitudinal (upper figure) or transverse (lower figure) impulsion for the CO-CSNW-8.8. The total energy is separated in the core and the shell at $2 \mathrm{THz}$ (first row of each subfigure) and $12 \mathrm{THz}$ for the longitudinal impulsion (second row of the upper figure) and $4 \mathrm{THz}$ for the transverse one (second row of the lower figure). The energy is normalized by the maximum value for the whole NW.

face (see Table IV). A sufficiently thick shell thus seems to shield the transport in the core from the effect of the free surface. Another mark of the interaction between the shell and the core can be seen in Fig. 10. The energy distribution in the core for the CO-CS-NW-8.8 in the LS direction after a transverse excitation does not follow a monotonous decrease from the impulsion origin to the end of the NW. On the contrary, it increases, confirming that there is an energy transfer from the shell into the core. The fact that this effect does not contribute to increase but to decrease the energy flux in the LS direction, must be due to strong resulting spatial heterogeneity, as already observed in Fig. 8 ,

At higher frequencies, here represented by $12 \mathrm{THz}$ in Fig. 10 for the $\mathrm{L}$ polarization and by $4 \mathrm{THz}$ for the $\mathrm{T}$ polarization, the behaviors in the SL and in the LS directions are much more similar. At these frequencies, there is a clear distinction between the diffusive peak and the propagative waves. The propagative part is limited to the core and a diffusive peak appears in the core and the shell in both directions. This is confirmed by the cross section view in Tables III to VI (rightmost columns), where the ballistic part, exiting the impulsion zone, takes an ellip- soidal shape limited to the inner core. This shape results from the combination of the time limited excitation and interfacial effects. The energy left behind by those propagating waves is dispersed evenly in the core section. The absence of irregularities on the WP path could explain why the MFP is higher than at lower frequencies where the interfaces continuously hinder the propagation. Moreover, for frequencies above $4 \mathrm{THz}$ all the geometries have similar MFP dependence on frequency for both longitudinal and transverse polarizations (see Fig. 6). Furthermore, there is the same amount of energy left and right of the impulsion (see Fig. 9), indicating that there is no apparent asymmetry in the energy transfer. Additionally, it seems that there is no important propagation of high-frequency phonons in the shell and the propagation in the core is no more affected by the shell thickness. However, if we focus on the diffusive transport, then we can see a slight asymmetry in the diffusive peak in the shell after a transverse excitation at $4 \mathrm{THz}$ (Fig. 10). This asymmetry is a qualitative visualisation of the higher diffusivity in the SL direction shown in Fig. 6 .

\section{DISCUSSION AND CONCLUSION}

A new strategy of thermal rectification is examined here, with an asymmetric core/shell nanowire and, as driving phenomenon, the interaction between the amorphous shell and crystalline core. The rectification estimated is of the order of $5 \%$ ( $90 \%$ for the case of crosssection selection on the hottest thermostat), indicating a better heat flow from the smaller part to the larger one. This finding is in contrast to some of the previous systems studied in the literature, as shown experimentally in nanoribbons 32] or for mass loaded graphene nanotube 31, and predicted for nanohorns 29] as well as for nanocones [30, but in agreement with others [39. Different explanations could be proposed for this discrepancy; it may be due, for example, to the fact that in the graphene study the MFP is much larger than the studied sample lengths. Phonons generated on the larger side reach ballistically the other side while modes generated on the smaller side tend to be scattered before reaching the other side due to edge effects [32, 69. For the Si NWs configurations studied here, most of the modes have a MFP lower than or comparable to the NWs length (see Fig. 6).

In our samples, the effect of the shell shape and the interfaces parallel to the heat flow on the spatial heterogeneity in the low-frequency ballistic transport of phonons and on the diffusive transport are the main causes of rectification. This is confirmed by the fact that effective medium models fails to predict the observed rectification. An example is the interesting model proposed by Peyrard et al. [12] based on the Fourier law where the flux is estimated thanks to an iterative method that uses $\kappa$ as a function of the temperature and the posi- 
tion from the imposed temperature gradient. This flow is then used to update the temperature profile. This new temperature profile allows updating $\kappa(x, T)$ for the next iteration. In our case the thermal conductivity $\kappa$ along the nanowire, assuming a common temperature gradient, and by simple summation of the thermal fluxes, could be approximated as

$$
\begin{aligned}
\kappa(x, T)= & \kappa_{a-S i}(T) \frac{V_{a-S i}(x)}{V_{a-S i}(x)+V_{c-S i}(x)} \\
& +\kappa_{c-S i}(T) \frac{V_{c-S i}(x)}{V_{a-S i}(x)+V_{c-S i}(x)}
\end{aligned}
$$

with $V_{a-S i}(x)$ and $V_{c-S i}(x)$ the respective volumes of the amorphous and crystalline phases as a function of the position and $\kappa_{a-S i}(T)$ and $\kappa_{c-S i}(T)$ the thermal conductivities of the different phases as a function of the temperature 70, 71. This model fails to predict the rectification observed for the CO-CS-NW-4.4 and CO-CS-NW8.8 , since it does not induce any directional dependence. However, rectification could be expected, since around $300 \mathrm{~K}$ the thermal conductivity of a-Si increases modestly with temperature while the $\kappa$ of c-Si decreases with temperature. This model would induce a smaller flow in the SL direction, when the small section (composed mainly of c-Si) is at high temperature and the larger part (where the a-Si dominates) is at lower temperature. However, with the NEMD simulations, we reported an opposite bias. The same remark holds for the diffusivity, that should decrease, based on a simple summation rule, in the direction where the amorphous fraction increases (SL). This is clearly not the case here. These two points indicate that existing models, which do not take into account interfaces, are not able to explain the thermal rectification in our systems. Heat transport directionality in our case results from the impact of the variable shell thickness on the behavior of phonon scattering at the crystalline/amorphous interface; thinner shell increases phonon scattering at these interfaces. Thus, the propagation of phonons depends on the propagation direction (see Table VI)

For the propagative transport, the larger wavelengths seem to be more influenced by the dimensional reduction and/or the proximity of the interface/free surface than lower wavelengths. This can explain the stronger dispersion in the smaller section of the CO-CS-NW-8.8 than in the larger section (central column of Tables VI and VI). The similarity between the pattern of displacement (left column) for the CO-CS-NW-8.8 in SL direction where the section is smaller and the one of the CL-CS-NW tends to validate the influence of the free surface on the propagation in the core through the shell. Moreover, at $2 \mathrm{THz}$ a surface wave appears, part of the energy seems to be confined at the surface during the WP propagation. The perturbation of long wavelength modes, localization at the free surface and their effect on the thermal conductivity in thin NWs is already well documented [72]. For the WP at $2 \mathrm{THz}$, a phase shift appears between the center of the core and the core-shell interface, as the wave propa- gates in the CL-CS-NW and CO-CS-NW-8.8 (Tables III to VI). However, this phase shift disappears in the SL direction for the CO-CS-NW-8.8. At low frequencies the wave can propagate in the core and the shell at a similar group velocity as in the absence of interface. In the larger section the wave packet seems to have mainly an ellipsoidal shape with a section larger than the core meaning that the amorphous/crystalline interface does not seem to affect much the propagation at this frequency. This implies that the free surface plays a more important role on the global propagation. Whereas at higher frequencies, for instance at $12 \mathrm{THz}$ (Tables III to VI right column), ballistic propagation happens only in the inner parts of core where the interfaces do not affect anymore the propagation.

The diffusive transport is also influenced by the presence of amorphous/crystalline interfaces. Indeed, a diffusivity comparable to the values found in the purely amorphous samples is measured even when the amount of amorphous shell is negligible (see Fig. 6), while a ballistic transport would be expected in the crystalline part. The presence of the amorphous/crystalline interfaces aligned with the direction of propagation is thus sufficient to induce a diffusive transport in the crystalline core as well. This strong influence of the shell on the diffusive transport has been already suggested here by the presence of a diffusive part in the core after a nonrandom excitation (see Fig. 10). For both the CO-CS-NW-4.4 and CO-CS-NW-8.8, the diffusivity is higher in the SL direction. Thus, the diffusivity seems to favor the observed rectification. However, the diffusive modes are not dominant on the heat transport, as shown in the Appendix B. It appears clearly in Fig. 13 that the propagative modes contribution dominates the heat transport in our systems. The diffusive modes account for less than $20 \%$ of the total thermal conductivity and they finally do not contribute to the rectification as can be seen from the juxtaposition of the SL and LS temperature dependence of the thermal conductivity.

The flux rectification obtained in our systems is lower compared to the results obtained for nanoribbons 32 or nanohorns 73, for example. However, it is systematically observed and can be obtained in simply designed samples, thanks to the originality of our new strategy of rectification. Moreover, the present configuration could be improved by texturing the amorphous/crystalline interface with a sawtooth pattern, which is known to promote rectification 35, or by using a cascade of nanowires in pyramidal shape, as proposed by Yang et al. [74. As the interfaces and surfaces are the driving forces for the rectification, their asymmetric roughness and atomic structure could enhance the observed thermal rectification.

In Sec. III the thermal rectification increases in increasing the distance between the two thermostats for a constant $\Delta T$ of $40 \mathrm{~K}$. This enhancement is surprising and contrasts with existing studies in which the rectification either decreases 38 or is invariable when the length is 
increased [39]. In the case of rectification due to interfacial effect, when the interface between the two materials is perpendicular to the heat flux, the rectification fades away as the length increases [38. In the CO-CS-NW case, the interface is parallel to the heat flux and this induces an opposite impact of the length on the rectification. The larger interfacial area seems to induce a higher rectification for the longer asymmetric nanowire.

In the case of larger $\Delta T(80 \mathrm{~K})$, we find a lower rectification for the same system. This is in contrast to previous systems where rectification was based only on geometrical effects [26, 34, 38, 73]. In the later studies, the rectification increases on increasing the temperature difference. We believe that this opposite behavior could be explained by the enhancement of the phonon-phonon scattering processes compared to the boundary/interface scattering [16, 29].

Other preliminary results show finally that the rectification does not increase for a lower mean temperature. This is in agreement with $\mathrm{Ju}$ et al. 39] who have shown that the rectification tends to decrease with temperature below $500 \mathrm{~K}$ for Si nanoribbons. The effect of length and temperature is thus not so trivial, since it may result from complex processes and there is no quantitative predictive explanation until now. Our article focuses on highlighting the rectification in a more qualitative manner.

Concerning the studied geometries: Constant diameter crystalline NWs embedded in a conical amorphous shell are not common. Nanowires can take a conical shape, as the result of the competition between catalyzed axial growth and uncatalyzed radial growth. In this case the resulting NW is fully crystalline. Alternatively, the oxidation could result in the formation of an oxide shell of uniform thickness. Nevertheless, cylindrical core/conical shell structures have been already experimentally obtained by Lin et al. in 2004 with a silicon carbide core and an amorphous silica shell 75 and by Huo et al. in $\mathrm{TiO}_{2}$ nanowire cores and carbon conical shells 76 . The latter elaboration examples show the possibility of realization of such asymmetric NWs to obtain thermal rectification.

The analysis of the WP simulation indicates that the low frequencies are crucial for the rectification. Another way to identify the modes responsible for the rectification would be to perform a spectral flux analysis, as proposed by Sääskilahti et al. [77. Such an analysis would allow us to identify the important frequencies in both SL and LS directions. However, due to the complexity of the geometries and the related calculation time it was not possible to realize such simulations in the scope of the current paper. But we propose in Appendix B an alternative way to get the frequency dependence of the thermal conductivity, together with the ratio of ballistic to diffusive contributions to the thermal conductivity. In this computation, the ballistic contribution is computed from the mean free path and the velocity of the wave packets, weighted by the vibrational density of states and the heat capacity of the sample, while the diffusive contribution involves the diffusivity weighted as well by the vibrational density of states and the heat capacity. It is shown, as discussed previously, that the diffusive contribution to the thermal conductivity is 4 times smaller than the ballistic one (contrary to what happens in purely amorphous samples or in samples with separated inclusions [42]) and that the rectification in the diffusive contribution is finally negligible.

To conclude, we have observed that nanowires with a crystalline core and an asymmetric amorphous silicon shell transports heat better in the small to large crosssection direction, in contrast to the majority of the existing literature results. The heat transport in the amorphous shell seems to be an important factor of the rectification, as only the configurations with the large amorphous fraction showed a significant rectification. The ballistic transport at low frequencies seems to be also an important factor of influence on the observed rectification. This transport is affected differently by the interfaces in the two directions, with strong scattering in the large to small cross-section direction. The transport in the amorphous shell, at the core/shell interface, and at the free surface of the shell are all important factors. The thickness of the shell affects the ballistic transport in the core. Nevertheless, the opening angle of the asymmetric amorphous shell does not seem to have a linear impact on the rectification; for small opening angles there is almost no rectification, while for angles $4.4^{\circ}$ and $8.8^{\circ}$ the heat flux rectification is constant and around 5\%. Finally, the shape of the shell is also an important factor; the telescopic NWs have a lower rectification than the conical CS-NWs. The conical shape increases the rectification, probably by favoring energy transfer from the shell to the core.

\section{Appendix A: Determination of the flux}

To compute the thermal flux in each direction we rely on the cumulative energy exchanged by the thermostats, as displayed in the first row of Figs. 11(a) and 11(b). First, in order to compute a reliable flux, the transient behavior due the setting up of the thermal gradient must be avoided. To this end the exchange rate is computed from $1000 \mathrm{ps}$, when the temperature profile is stabilized until the end of the simulation. The slope of the cumulative exchanged energy by the thermostat during steady state gives the flux imposed by this thermostat. This slope is computed thanks to a least-squares fit to a linear function. This power exchanged is displayed in the second row of Fig. 11(a) and 11(b), the symbols representing the average slope over five runs, with the error bars corresponding to the dispersion. For the computation of the rectification, the flux (or power exchange) in the SL case (solid lines) is compared to the LS case (dashed lines). For this we computed the powered exchange at each thermostat (second row of Fig. 11). However, in spite of a stable temperature profile, the power 
exchanged at the cold thermostat (in blue) is higher than at the hot thermostat (in red), for both the SL and LS cases, represented respectively by circles and triangles in Fig. 11). This means that more energy is retrieved than added, in spite of the stable temperature profile. This is true for the CL-CS-NW [Fig. 11(a)] and the CO-CS-NW [Fig. 11(b)], thus this is not directly caused by the asymmetric shape of the sample. In order to have a single representative value for both thermostats, we consider the flux as the average between the power exchanged at the cold and hot thermostats (see the third row of Fig. 11). Finally, to compute the thermal rectification, we compare this value for the SL and LS direction as in Eq. (1). The power exchanged in the SL case is systematically higher than in the LS case, for the geometries inducing rectification like the CO-CS-NW-8.8 in Fig. 11, indicating that the rectification direction is not sensitive to the choice of the (cold or hot) thermostat where the measurement is made.

It is also to be noted that the thermostats used are thin, to confirm the validity of these results, another thermostat configuration is tested for the CO-CS-NW-4.4. For these tests the heat bath are extended and placed in prolongations of the NWs, that is a prolongation crystalline core one side and a cylindrical prolongation composed of shell of radius $R_{\text {Shell }}$ in the other. This configuration gives similar results in terms of rectification.

\section{Appendix B: Estimation of $\kappa_{d i f f}$ and $\kappa_{\text {prop }}$}

To evaluate the relative importance of the propagative and diffusive part of the thermal conductivity the method described by Tlili et al. 42 is used. The contribution of the propagative modes is estimated by the usual expression:

$$
\kappa_{\text {prop }}=\int_{0}^{\nu_{\max }} \frac{m}{3} C(\nu, T) v(\nu) l(\nu) g(\nu) d \nu
$$

with $C(\nu, T)$ the heat capacity at the temperature $T$ and frequency $\nu, v$ the group velocity, $l$ the mean free path, $g$ and $m$ density of state and degree of freedom associated to the polarization. $\nu_{\max }$ is the frequency for which the group velocity is zero.

The contribution of the diffusive modes is estimated with:

$$
\kappa_{\text {diff }}=\int_{0}^{\infty} 3 C(\nu, T) g(\nu) D(\nu) d \nu
$$

with $D$ the diffusivity and $g$ the global density of states computed in the main body of the article.

The computation of $l(\nu), g(\nu)$, and $D(\nu)$ are detailed in the main body of the article. The group velocity and the density of state associated to the different polarizations remain to be computed. These missing quantities are estimated thanks to the dynamical structure factor obtained from a spatial and temporal Fourier transform of the particles displacement. The dynamical structure factor as described by Damart et al. 78, is computed as follows:

$S(\mathbf{q}, \omega)=\frac{2}{N T}\left|\sum_{i}^{N_{a t}} \exp \left(-i \mathbf{q} \cdot \mathbf{r}_{\mathbf{i}}\right) \int_{0}^{\tau} \mathbf{u}_{\mathbf{i}}(t) \cdot \mathbf{m}_{\mathbf{q}} \exp (i \omega t) d t\right|^{2}$

with $\mathbf{q}$ the wave vector, $\mathbf{u}_{\mathbf{i}}$ and $\mathbf{r}_{\mathbf{i}}$ the displacement and position of the $i^{\text {th }}$ atom, $\mathbf{m}_{\mathbf{q}}$ the polarization vector (parallel or perpendicular to $\mathbf{q}$ ), $T$ the temperature, and $N$ the total number of atoms.

An example of $S(\mathbf{q}, \omega)$ for the longitudinal (a) and transverse (b) polarizations for a wave vector corresponding to the direction $\langle 100\rangle$ is displayed in Fig. 12. The phononic dispersion curves can already be distinguished, they are formally estimated as follows: To each wave vector is associated the frequency for which $S(\mathbf{q}, \omega)$ has the highest value within the acoustic phonons frequency range. The dispersion obtained is then fitted to a sinus function, whose derivative can be used to compute the group velocity. The dynamical structure factor can also provide an estimation of the transverse and longitudinal DOS by an integration over all the wave vector.

The different contributions [Eq. (B1) and Eq. (B2)] to the thermal conductivity as a function of temperature are represented in Fig. 13 (left). It appears clearly that the propagative modes contribution dominates the heat transport. The diffusive modes account for less than $20 \%$ of the total thermal conductivity and they do not contribute to the rectification as it can be seen from the SL and LS juxtaposition. The relative contributions to $\kappa$ as a function of the frequency at $300 \mathrm{~K}$ in the left panel of Fig. 13. shows that $\kappa_{\text {prop }}$ is higher than $\kappa_{\text {diff }}$ for all frequencies. It also appears that the peak in MFP around $10 \mathrm{THz}$ has a strong effect on the thermal conductivity. Note that the diffusive contribution, even if negligible in the case of CO-CS-NW, is notably large, since it is close to the diffusivity in amorphous silicon. The difference with the results of Tlili et al. for fully amorphous Si comes from the propagative part proportionally higher in our case 42 .

\section{Appendix C: Cross section view of the evolution of the wavepackets}

This section contains the Tables III to VI showing the propagation of longitudinal and transverse wave packets for impulsion at 2 and $12 \mathrm{THz}$, and 2 and $4 \mathrm{THz}$ respectively. The case of two representative configuration the CL-CS-NW and CO-CS-NW-8.8 are chosen. For the lowest-frequency both the displacement and the atomic kinetic energy are represented by a color scale, for the highest-frequency only the latter is represented. These tables are described in Sec. V. 

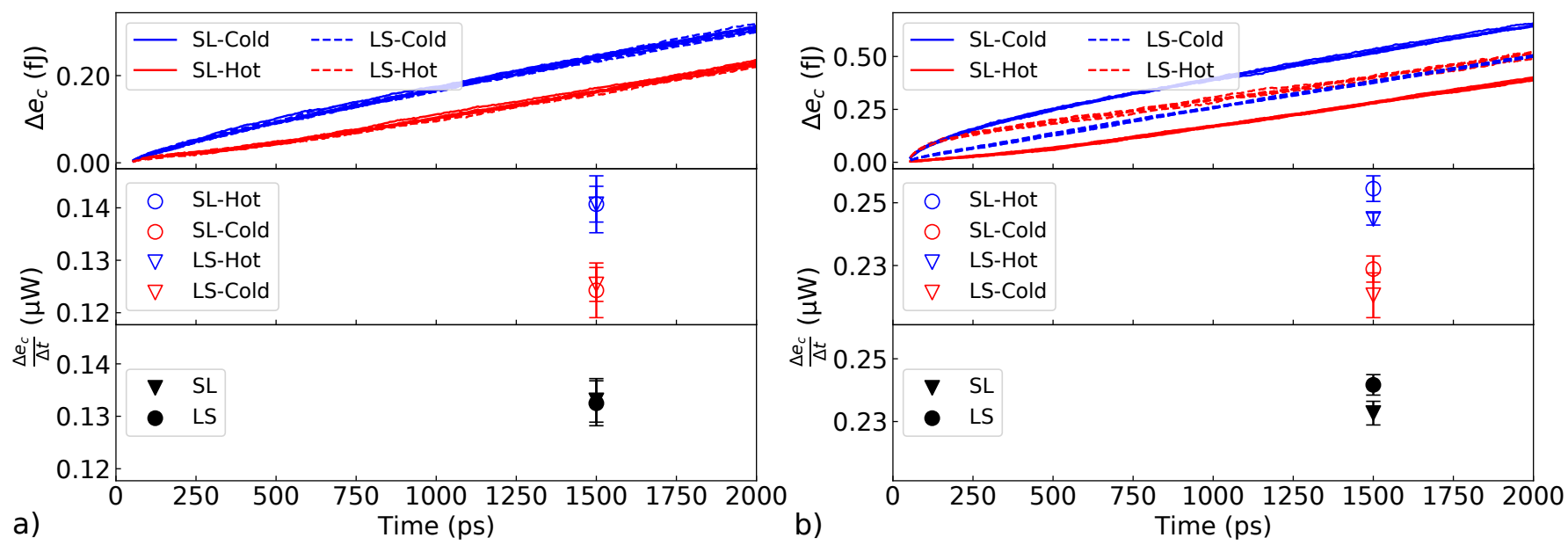

FIG. 11. Energy exchanged by the thermostats, for the CL-CS-NW and the CO-CS-NW-8.8: The first row represents the energy exchanged at each thermostat for five runs, the blue lines represent the energy retrieved by the cold thermostats, and the red lines the energy injected by the hot thermostats. The dashed lines distinguish the SL configuration from the LS configuration represented by the continuous line. The second rows represent the estimation of the energy flux, computed 1000 to $2000 \mathrm{ps}$, the red symbols represent the hot thermostats, the blue symbols represent the cold thermostats. In the third row the black symbols represent flux taken as the average between hot and cold thermostat. The inverted triangles represent the LS case and the crosses the SL.

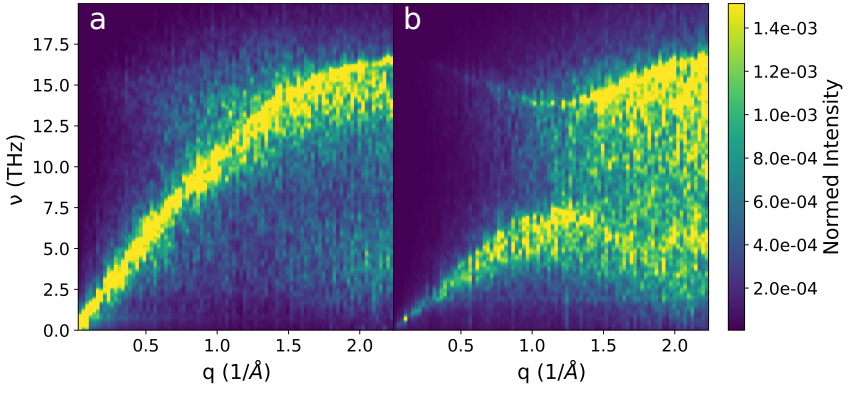

FIG. 12. $\quad S(\mathbf{q}, \omega)$ for (a) longitudinal and (b) transverse polarization in the $\Gamma X$ direction for the CO-CS-NW-4.4 computed with Eq. B3 and convoluted with a typical energy resolution curve of linewidth $0.33 \mathrm{THz}$ to smooth the obtained distribution 42 .
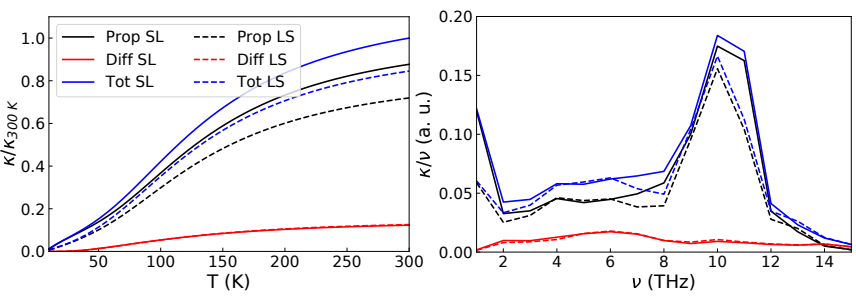

FIG. 13. Thermal conductivity of propagative modes $\kappa_{\text {prop }}$ (in black), and thermal conductivity of the diffusive modes $\kappa_{\text {diff }}$ (in red) and total thermal conductivity $\kappa_{\text {prop }}+\kappa_{\text {diff }}$ (in blue) for the CS-NW-CO-4.4, as a function of temperature in the left panel and contribution of the different frequencies at $300 \mathrm{~K}$ in the right panel. The dashed and plain lines represents respectively the LS and SL directions.

\section{ACKNOWLEDGMENTS}

This work was granted access to the HPC resources of IDRIS under the allocation 2020-A0070911092 made by GENCI. This work was made possible thanks to a Ph.D. scholarship accorded by the French Ministry of Education and Higher Education. We also thank the engineering federation Ingelyse for its financial support. Finally, we thanks V. Giordano and O. Bourgeois for fruitful discussions.
[1] J. A. Fleming, Proceedings of the Physical Society of London 14, 187 (1895).

[2] J. Bardeen and W. H. Brattain, Phys. Rev. 74, 230 (1948)
[3] M. Bass, P. A. Franken, J. F. Ward, and G. Weinreich, Phys. Rev. Lett. 9, 446 (1962)

[4] M. Maldovan, Nature 503, 209 (2013).

[5] S. Slussarenko and G. J. Pryde, Applied Physics Reviews 6, 041303 (2019), https://doi.org/10.1063/1.5115814 
[6] G. Wehmeyer, T. Yabuki, C. Monachon, J. Wu, and C. Dames, Applied Physics Reviews 4, 041304 (2017), https://doi.org/10.1063/1.5001072

[7] M. J. Martínez-Pérez, A. Fornieri, and F. Giazotto, Nature nanotechnology 10, 303 (2015).

[8] C. Starr, Physics 7, 15 (1936), https://doi.org/10.1063/1.1745338

[9] K. Ito, K. Nishikawa, H. Iizuka, and H. Toshiyoshi, Applied Physics Letters 105, 253503 (2014), https://doi.org/10.1063/1.4905132.

[10] M. Terraneo, M. Peyrard, and G. Casati, Phys. Rev. Lett. 88, $094302(2002)$.

[11] B. Li, L. Wang, and G. Casati, Phys. Rev. Lett. 93, 184301 (2004)

[12] M. Peyrard, Europhysics Letters (EPL) 76, 49 (2006).

[13] N. Roberts and D. Walker, International Journal of Thermal Sciences 50,648 (2011)

[14] H. Liu, H. Wang, and X. Zhang, Applied Sciences 9, 344 (2019).

[15] C. Dames, Journal of Heat Transfer 131, $10.1115 / 1.3089552 \quad$ (2009), 061301, https://asmedigitalcollection.asme.org/heattransfer/articlepdf $/ 131 / 6 / 061301 / 5621798 / 061301 \_1 . p d f$.

[16] Q.-X. Pei, Y.-W. Zhang, Z.-D. Sha, and V. B. Shenoy, Applied Physics Letters 100, 101901 (2012) https://doi.org/10.1063/1.3692173.

[17] X. Zhang, M. Hu, and D. Tang, Journal of Applied Physics 113, 194307 (2013) https://doi.org/10.1063/1.4804071.

[18] W. Kobayashi, Y. Teraoka, and I. Terasaki, Applied Physics Letters 95, 171905 (2009), https://doi.org/10.1063/1.3253712.

[19] D. Sawaki, W. Kobayashi, Y. Moritomo, and I. Terasaki, Applied Physics Letters 98, 081915 (2011), https://doi.org/10.1063/1.3559615

[20] M. López-Suárez, M. Royo, and R. Rurali, Phys. Rev. Materials 2, 113001(R) (2018).

[21] K. R. Hahn, M. Puligheddu, and L. Colombo, Phys. Rev. B 91, 195313 (2015)

[22] R. Rurali, X. Cartoixà, and L. Colombo, Phys. Rev. B 90, 041408(R) (2014)

[23] M. Hu, P. Keblinski, and B. Li, Applied Physics Letters 92, 211908 (2008), https://doi.org/10.1063/1.2937834.

[24] D. Li, Y. Wu, P. Kim, L. Shi, P. Yang, and A. Majumdar, Applied Physics Letters 83, 2934 (2003), https://doi.org/10.1063/1.1616981

[25] S. Hu, Z. Zhang, P. Jiang, J. Chen, S. Volz, M. Nomura, and B. Li, The journal of physical chemistry letters $\mathbf{9}$, 3959 (2018).

[26] Y. Wang, A. Vallabhaneni, J. Hu, B. Qiu, Y. P. Chen, and X. Ruan, Nano letters 14, 592 (2014).

[27] S. Ju and X. Liang, Journal of Applied Physics 112, 054312 (2012), https://doi.org/10.1063/1.4749394.

[28] G. Wu and B. Li, Phys. Rev. B 76, 085424 (2007)

[29] N. Yang, G. Zhang, and B. Li, Applied Physics Letters 93, 243111 (2008), https://doi.org/10.1063/1.3049603

[30] Z. Zhang, Y. Chen, Y. Xie, and S. Zhang, Applied Thermal Engineering 102, 1075 (2016)

[31] C. W. Chang, D. Okawa, A. Majumdar, and A. Zettl, Science 314, 1121 (2006), https://science.sciencemag.org/content/314/5802/1121.ful .p

[32] H. Wang, S. Hu, K. Takahashi, X. Zhang, H. Takamatsu, and J. Chen, Nature communications 8, 1 (2017).
[33] M. Kasprzak, M. Sledzinska, K. Zaleski, I. Iatsunskyi, F. Alzina, S. Volz, C. M. S. Torres, and B. Graczykowski, Nano Energy 78, 105261 (2020).

[34] X. Cartoixà, L. Colombo, and R. Rurali, Nano letters 15, 8255 (2015).

[35] Phonon Transport in Asymmetric Sawtooth Nanowires, ASME/JSME Thermal Engineering Joint Conference, Vol. ASME/JSME 2011 8th Thermal Engineering Joint Conference (2011) t30053, https://asmedigitalcollection.asme.org/AJTEC/proceedingspdf/AJTEC2011/38921/T30053/4600781/t30053_1.pdf.

[36] R. Dettori, C. Melis, R. Rurali, and L. Colombo, Journal of Applied Physics 119, 215102 (2016) https://doi.org/10.1063/1.4953142.

[37] M. Tovar-Padilla, L. Licea-Jimenez, S. A. Prez-Garcia, and J. Alvarez-Quintana, Applied Physics Letters 107, 084103 (2015), https://doi.org/10.1063/1.4929586

[38] N. Yang, G. Zhang, and B. Li, Applied Physics Letters 95, 033107 (2009), https://doi.org/10.1063/1.3183587

[39] S. Ju and X. Liang, Journal of Applied Physics 112, 024307 (2012), https://doi.org/10.1063/1.4737585

[40] K. Gordiz and A. Henry, Journal of Applied Physics 121, 025102 (2017), https://doi.org/10.1063/1.4973573

[41] L. Yang, B. Latour, and A. J. Minnich, Phys. Rev. B 97, 205306 (2018)

[42] A. Tlili, V. M. Giordano, Y. M. Beltukov, P. Desmarchelier, S. Merabia, and A. Tanguy, Nanoscale 11, 21502 (2019)

[43] B. Deng, A. Chernatynskiy, M. Khafizov, D. H. Hurley, and S. R. Phillpot, Journal of Applied Physics 115, 084910 (2014), https://doi.org/10.1063/1.4867047

[44] P. Desmarchelier, K. Termentzidis, and A. Tanguy, Semiconductor Science and Technology 35, 094001 (2020)

[45] H. Kim and V. Tomar, physica sta$\begin{array}{lllll}\text { tus solidi } & \text { (a) } & \mathbf{2 0 8}, & 2115 & \text { (2011), }\end{array}$ https://onlinelibrary.wiley.com/doi/pdf/10.1002/pssa.201026578

[46] B. Lee and R. E. Rudd, Phys. Rev. B 75, 195328 (2007)

[47] H.-P. Li and R.-Q. Zhang, Chinese Physics B 27, 036801 (2018).

[48] A. Malhotra and M. Maldovan, Scientific reports 6, 1 (2016).

[49] Y. Wang, B. Li, and G. Xie, RSC Adv. 3, 26074 (2013)

[50] J. Chen, G. Zhang, and B. Li, The Journal of Chemical Physics 135, 104508 (2011) https://doi.org/10.1063/1.3637044.

[51] D. Donadio and G. Galli, Phys. Rev. Lett. 102, 195901 (2009)

[52] T. Zushi, K. Ohmori, K. Yamada, and T. Watanabe, Phys. Rev. B 91, 115308 (2015)

[53] E. Blandre, L. Chaput, S. Merabia, D. Lacroix, and K. Termentzidis, Phys. Rev. B 91, 115404 (2015)

[54] L. Zhang, J.-S. Wang, and B. Li, Phys. Rev. B 81, 100301(R) (2010)

[55] A. France-Lanord, E. Blandre, T. Albaret, S. Merabia, D. Lacroix, and K. Termentzidis, Journal of Physics: Condensed Matter 26, 055011 (2014).

[56] A. Stukowski, Modelling and Simulation in Materials Science and Engineering 18, 015012 (2009)

[57] S. Plimpton, Journal of Computational Physics 117, 1 (1995)

F3] R. Vink, G. Barkema, W. van der Weg, and N. Mousseau, Journal of Non-Crystalline Solids 282, 248 (2001).

[59] Y. M. Beltukov, C. Fusco, D. A. Parshin, and A. Tanguy, Phys. Rev. E 93, 023006 (2016) 
[60] Y. M. Beltukov, V. I. Kozub, and D. A. Parshin, Phys. Rev. B 87, 134203 (2013).

[61] A. Savitzky and M. J. Golay, Analytical chemistry 36, 1627 (1964).

[62] M. T. Dove and M. T. Dove, Introduction to Lattice Dynamics, Vol. 4 (Cambridge University Press, 1993).

[63] M. Verdier, D. Lacroix, and K. Termentzidis, Journal of Applied Physics 126, 164305 (2019), https://doi.org/10.1063/1.5108618.

[64] P. K. Schelling, S. R. Phillpot, and P. Keblinski, Phys. Rev. B 65, 144306 (2002).

[65] Y. Zhou, X. Zhang, and M. Hu, Phys. Rev. B 92, 195204 (2015)

[66] Y. M. Beltukov, D. A. Parshin, V. M. Giordano, and A. Tanguy, Phys. Rev. E 98, 023005 (2018)

[67] G. Xie, Y. Guo, X. Wei, K. Zhang, L. Sun, J. Zhong, G. Zhang, and Y.-W. Zhang, Applied Physics Letters 104, 233901 (2014), https://doi.org/10.1063/1.4882083.

[68] S. Xiong, K. Sääskilahti, Y. A. Kosevich, H. Han, D. Donadio, and S. Volz, Phys. Rev. Lett. 117, 025503 (2016)

[69] Y. Wang, S. Chen, and X. Ruan, Applied Physics Letters 100, 163101 (2012), https://doi.org/10.1063/1.3703756.
[70] P. B. Allen, J. L. Feldman, J. Fabian, and F. Wooten, Philosophical Magazine B 79, 1715 (1999), https://doi.org/10.1080/13642819908223054

[71] C. J. Glassbrenner and G. A. Slack, Phys. Rev. 134, A1058 (1964).

[72] R. Rurali, Rev. Mod. Phys. 82, 427 (2010).

[73] G. Wu and B. Li, Journal of Physics: Condensed Matter 20, 175211 (2008).

[74] X. Yang, D. Yu, B. Cao, and A. C. To, ACS applied materials \& interfaces 9, 29 (2017).

[75] M. Lin, K. P. Loh, C. Boothroyd, and A. Du, Applied Physics Letters 85, 5388 (2004), https://doi.org/10.1063/1.1828601.

[76] K. Huo, X. Zhang, L. Hu, X. Sun, J. Fu, and P. K. Chu, Applied Physics Letters 93, 013105 (2008), https://doi.org/10.1063/1.2955519

[77] K. Sääskilahti, J. Oksanen, S. Volz, and J. Tulkki, Phys. Rev. B 91, 115426 (2015).

[78] T. Damart, V. M. Giordano, and A. Tanguy, Phys. Rev. B 92, 094201 (2015) 
TABLE III. Cross-sectional view of wave-packet evolution after a transverse impulsion in the CL-CS-NW. Left column: Atomic displacement in the polarization direction with an impulsion at $2 \mathrm{THz}$. Middle column: The atomic kinetic energy at 2 THz. Right column: The atomic kinetic energy at $4 \mathrm{THz}$.

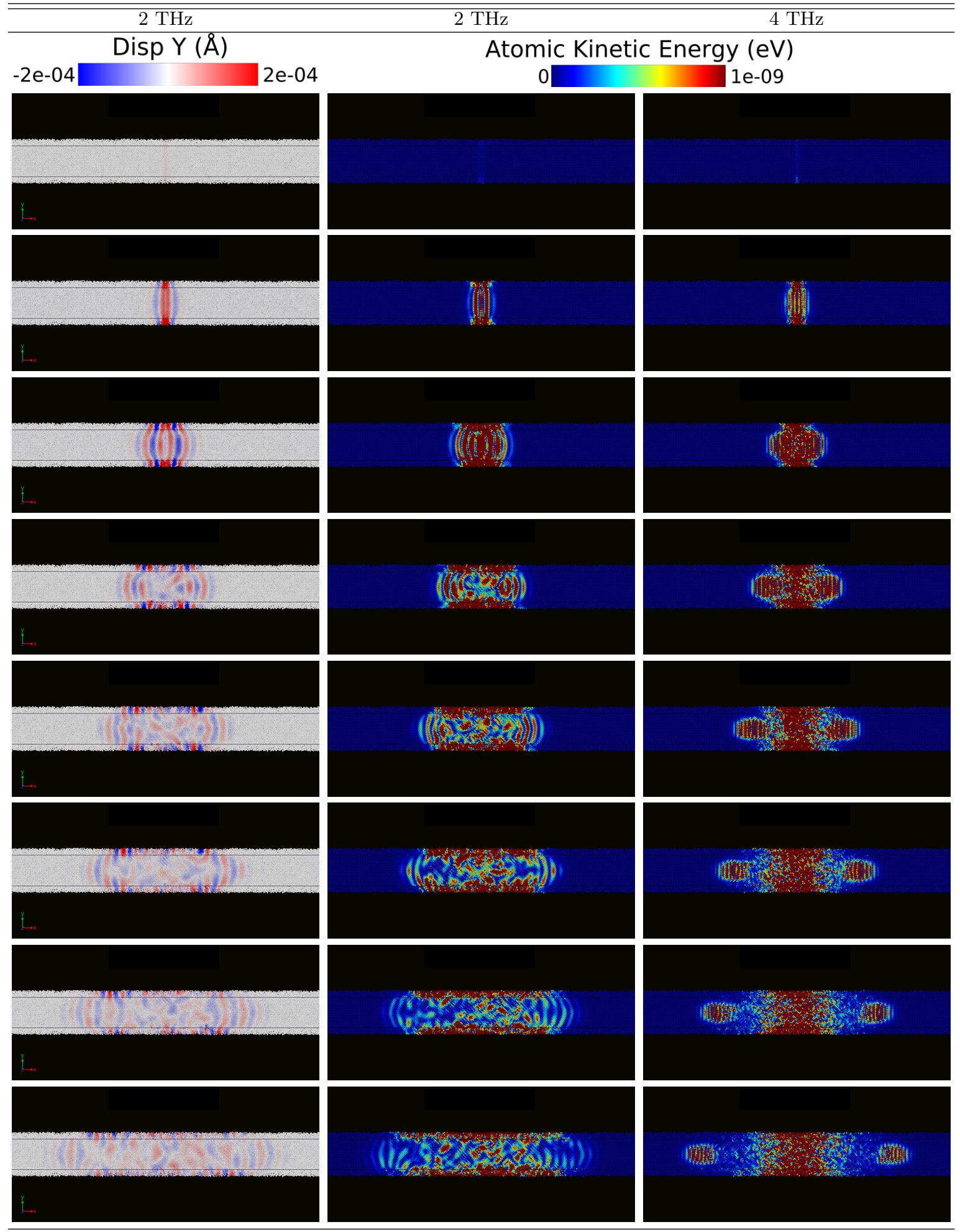


TABLE IV. Cross-sectional view of wave-packet evolution after a longitudinal excitation in the CL-CS-NW. Left column: Aatomic displacement in the polarization direction with an impulsion at $2 \mathrm{THz}$. Middle column: The atomic kinetic energy at $2 \mathrm{THz}$. Right column: The atomic kinetic energy at $12 \mathrm{THz}$

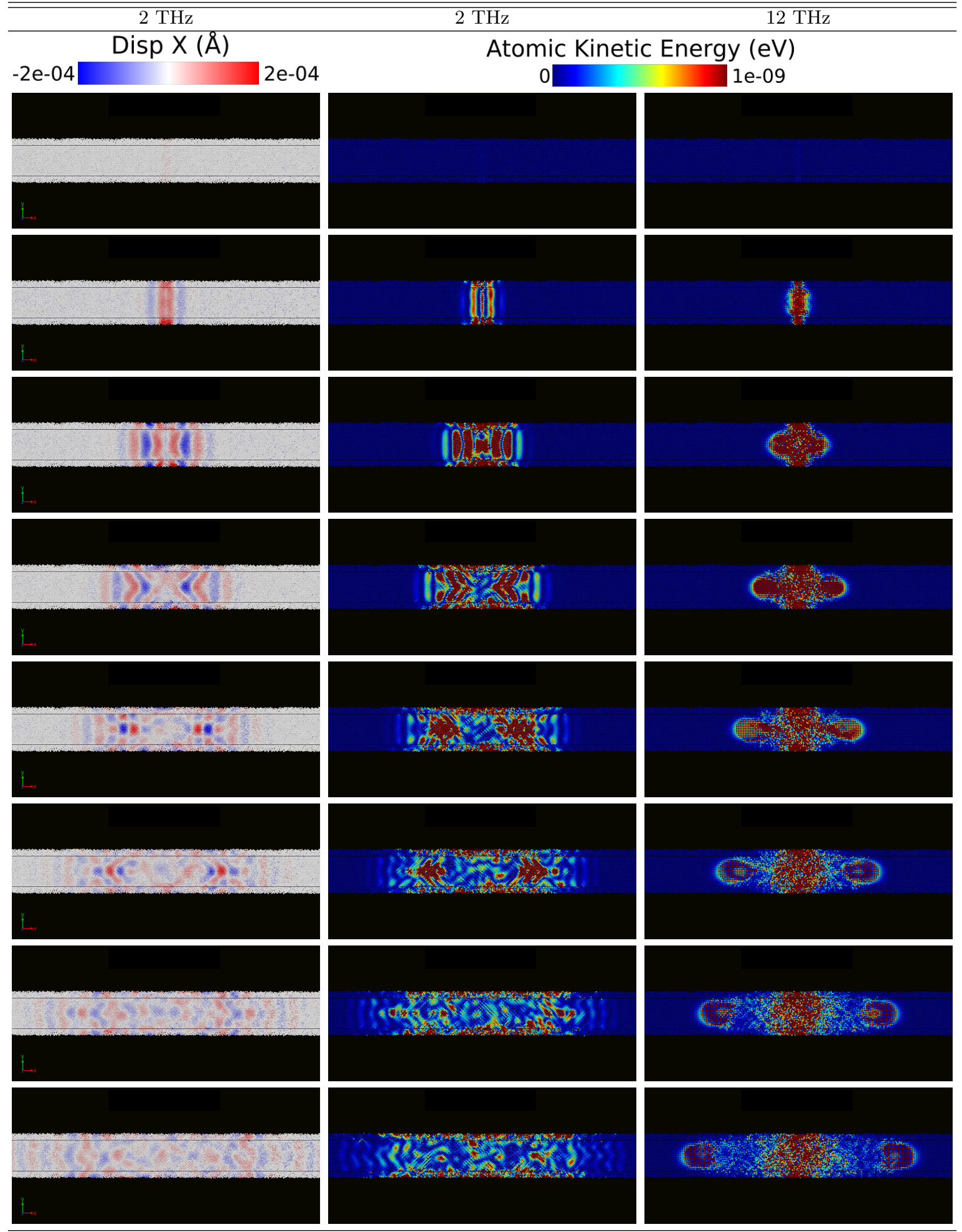


TABLE V. Cross-sectional view of wave-packet evolution after a transverse excitation in the CO-CS-NW-8.8. Left column: Atomic displacement in the polarization direction with an impulsion at $2 \mathrm{THz}$. Middle column: The atomic kinetic energy at $2 \mathrm{THz}$. Right column: The atomic kinetic energy at $4 \mathrm{THz}$.

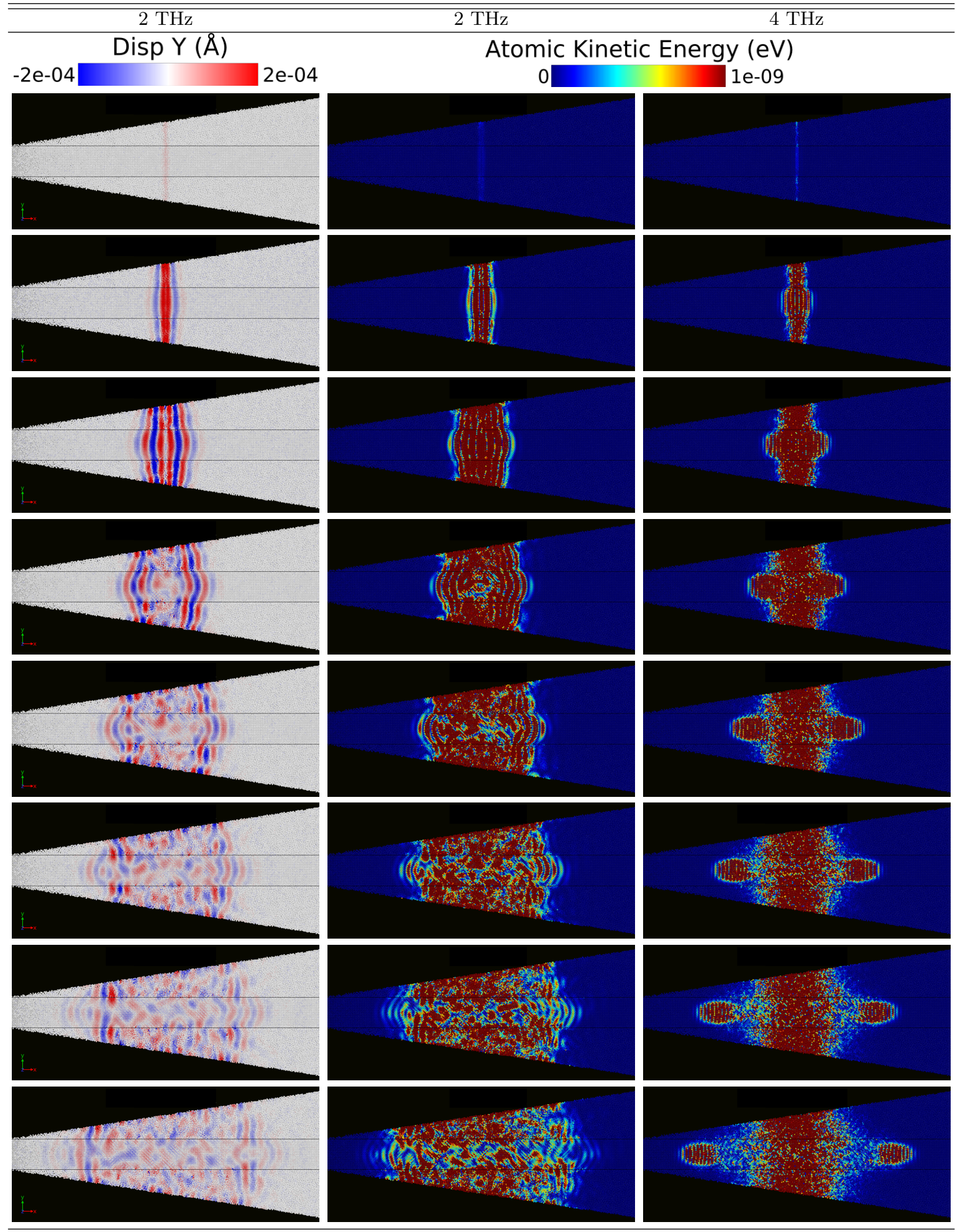


TABLE VI. TABLE VI. Cross-sectional view of wave-packet evolution after a longitudinal excitation in the CO-CS-NW-8.8. Left column: Atomic displacement in the polarization direction with an impulsion at 2 THz. Middle column: The atomic kinetic energy at $2 \mathrm{THz}$. Right column: The atomic kinetic energy at $4 \mathrm{THz}$

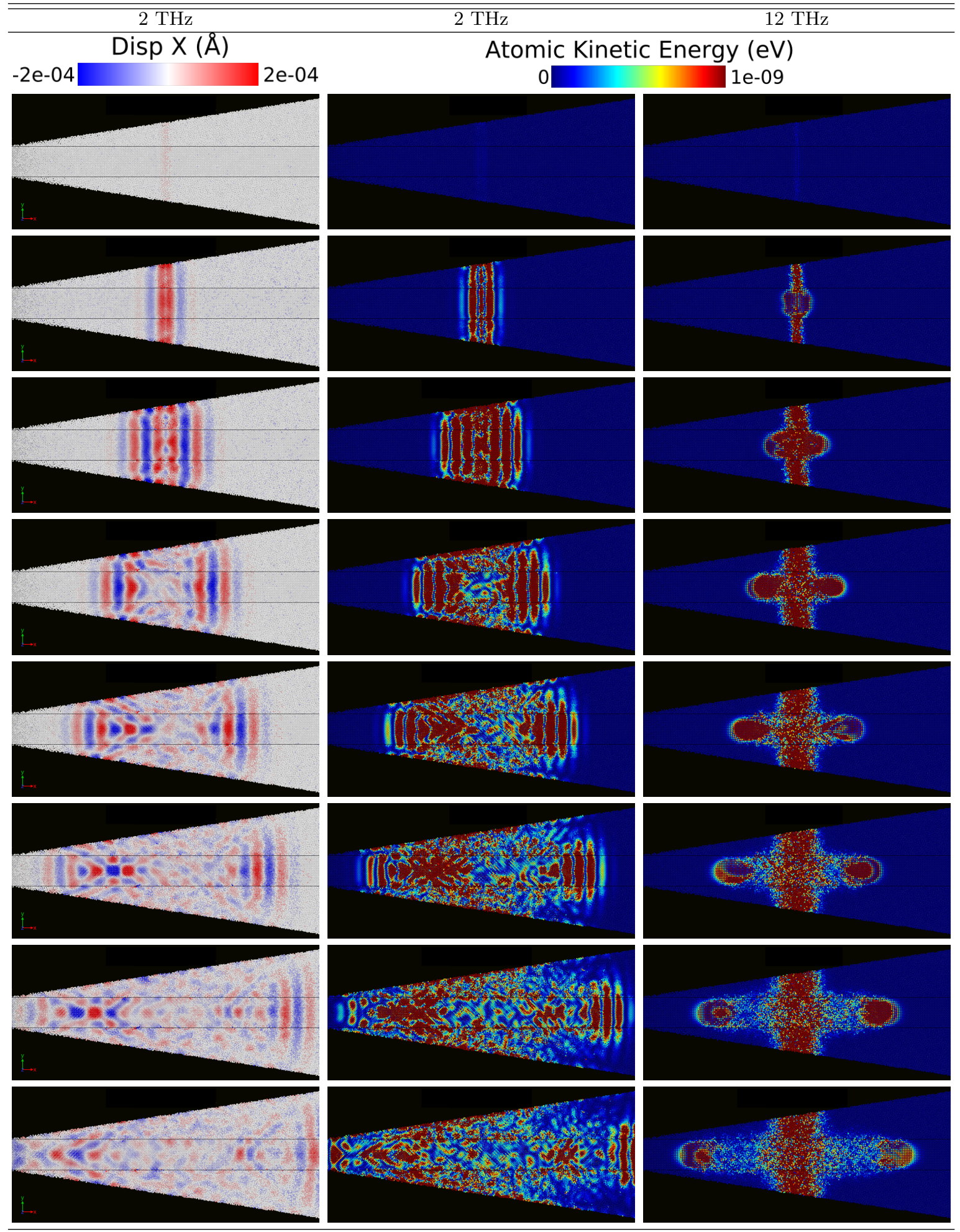

